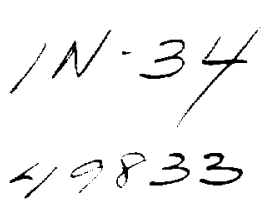

NASA Contractor Report 189184

ICOMP-95-7

\title{
Nonlinear Stability of Non-Stationary Cross-Flow Vortices in Compressible Boundary Layers
}

\author{
J.S.B. Gajjar
}

Institute for Computational Mechanics in Propulsion

Lewis Research Center

Cleveland, Ohio

and The University of Manchester

Manchester, England

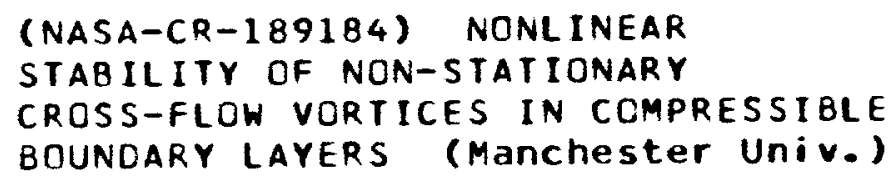

Prepared for

Lewis Research Center

Under Cooperative Agreement NCC3-370

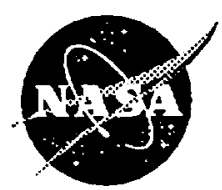

National Aeronautics and

Space Administration

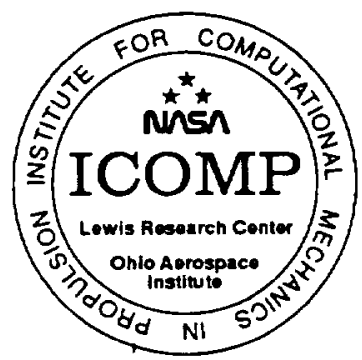




\title{
NONLINEAR STABILITY OF NON-STATIONARY CROSS-FLOW \\ VORTICES IN COMPRESSIBLE BOUNDARY LAYERS
}

\author{
J.S.B. Gajjar \\ Institute for Computational Mechanics in Propulsion \\ Lewis Research Center \\ Cleveland, Ohio 44135 \\ and The University of Manchester \\ Department of Mathematics \\ Oxford Road \\ Manchester M13 9PL England
}

\begin{abstract}
The nonlinear evolution of long wavelength non-stationary cross-flow vortices in a compressible boundary layer is investigated and the work extends that of Gajjar (1994) to flows involving multiple critical layers. The basic flow profile considered in this paper is that appropriate for a fully three-dimensional boundary layer with $O(1)$ Mach number and with wall heating or cooling. The governing equations for the evolution of the cross-flow vortex are obtained and some special cases are discussed. One special case includes linear theory where exact analytic expressions for the growth rate of the vortices are obtained. Another special case is a generalization of the Bassom \& Gajjar (1988) results for neutral waves to compressible flows. The viscous correction to the growth rate is derived and it is shown how the unsteady nonlinear critical layer structure merges with that for a Haberman type of viscous critical layer.
\end{abstract}




\section{Introduction}

The transition to turbulence of fluid flows has aroused considerable interest in recent years and substantial progress has now been made towards understanding the early stages of transition in many shear and planar boundary layer flows. The use of self-consistent asymptotic analysis in conjunction with the correct description of the flow structure in the limit of large Reynolds numbers, has given a remarkable insight into the delicate balances and different processes operating in a transitional boundary layer. There are many routes to transition in a boundary layer. Carefully controlled experiments with predominantly two-dimensional disturbances have highlighted the role played by disturbance three-dimensionality, Nishioka et al (1979), Klebanoff et al (1962), Kachanov \& Levchenko (1984), Corke \& Mangano (1989). After an initial stage in which the two-dimensional disturbances dominate, the onset of three-dimensionality leads rapidly to more complex structures and nonlinear interactions leading ultimately to turbulence. Much of this initial linear phase and some of the subsequent complicated nonlinear stage can now be described, if not quantitatively at least in a convincing qualitative manner using asymptotic theories, see for example the papers by Goldstein \& Choi $(1989,1990)$, Hall \& Smith (1991), Goldstein \& Lee (1992), Wu et al (1993,1994), Smith, \& Stewart (1987), Stewart \& Smith (1992), Smith \& Bowles (1992), Hultgren (1992), Goldstein (1994).

Our concern here is with the stability of fully three-dimensional boundary layers rather than two-dimensional boundary layer flows. One of the main differences between the stability of two and three-dimensional incompressible boundary layers is the occurrence of cross-flow instability in the latter. Whereas a typical two-dimensional incompressible boundary layer is not subject to inviscid instability, a three-dimensional boundary layer almost invariably is. The mechanism was first explained by Stuart in Gregory, Stuart \& Walker (1955) and he showed that in certain flow directions the velocity profile could be inflexional and hence subject to Rayleigh instability. This instability is known as cross-flow instability. A two-dimensional compressible boundary layer is also subject to inviscid instability because of the generalized inflexion point, Lees \& Lin (1946), Mack (1984, 1986). However cross-flow instability is believed to be more important even for compressible flows, especially at low Mach numbers, because of the much larger growth rates. Cross-flow instability is the main cause of transition of several flows of aerodynamic importance such as the flow past swept aircraft wings and the flow near the attachment line. See for instance the reviews of three-dimensional boundary layer instability by Reed \& Saric (1989) and Arnal (1986). Despite its importance cross-flow instability has not received as much theoretical attention as the planar case as it deserves.

Many experiments have been performed to study cross-flow instability. The flow over a rotating disk has been widely used as a prototype of a fully three-dimensional boundary layer flow and has been studied experimentally by Gregory, Stuart \& Walker (1955), 
Wilkinson \& Malik (1983) amongst others. The most commonly observed manifestation of cross-flow instability is the formation of a pattern of equi-spaced co-rotating stationary vortices. The stationary cross-flow instability arises from the amplification of zero frequency waves in the boundary layer. A non-stationary, or a travelling wave, pattern of cross-flow vortices is also possible. In experiments on swept-wing flows Bippes\& NitchkeKowsky(1987), Muller \& Bippes (1988), Kohama et al (1991), both types of instability have been found to occur. Poll (1985) has also observed both types of instability in experiments with swept cylinders. Muller \& Bippes (1988) have also investigated nonlinear effects and found that both the stationary and the non-stationary vortices reach a nonlinear equilibrium amplitude. In the experiments of Kohama et al (1991) an explosive secondary instability was seen prior to the onset of transition to turbulence.

Theoretical investigations of the linear stability of cross-flow vortices have to date been largely confined to incompressible flows. The stability properties of stationary crossflow vortices were first investigated by Gregory, Stuart \& Walker (1955). Malik(1986) and Balakumar \& Malik(1990) have computed neutral and growth rate curves for both the stationary and travelling wave disturbances for the rotating disk. Hall (1986) computed the corrections to the neutral values for the stationary case in the inviscid limit and also obtained the structure for the zero shear stress mode discovered by Malik (1986). Bassom \& Gajjar (1988) have looked at various linear and nonlinear aspects of the neutral stability of non-stationary cross-flow vortices. There have been various studies based on numerical simulation of either the full Navier-Stokes equations, Meyer \& Kleiser (1988) or an approximate form such as parabolised stability equations approach of Malik \& $\mathrm{Li}(1992)$, Malik it et al. (1994). The numerical simulations show the nonlinear saturation of crossflow vortices which is in qualitative agreement with the experiments of Muller \& Bippes (1988).

An objective of the present work is to study the nonlinear evolution of non-stationary cross-flow vortices in compressible boundary layers using unsteady nonlinear critical layer theory. The latter has been successfully used in the studies of the instability of many planar boundary layer and shear flows, see for instance the papers cited earlier. Excellent reviews of the early ideas on nonlinear critical layers are given in Stewartson (1981) and Maslowe (1986), and more recent work is surveyed in Goldstein (1994) and Cowley \& Wu (1994). The assumptions and scalings used here lead to a fully nonlinear partial differential system for the evolution of the vortex as in Goldstein, Durbin \& Leib (1987), Goldstein \& Leib (1988), Goldstein \& Hultgren (1988), Goldstein \& Wundrow (1990), Gajjar (1994), and not an integro-differential equation of the Hickernell (1984) type. The scalings adopted are appropriate for low frequency long wavelength cross-flow vortices which have small growth rates. For shorter waves the weakly nonlinear approach leading to a Hickernell type amplitude equation is more appropriate, Gajjar (1995). Gajjar (1994) has studied the 
development of long wavelength oblique modes on two-dimensional compressible boundary layers and the present work extends this to three-dimensional boundary layers. The bulk of the analysis can be simplified through the introduction of appropriate Squire coordinates and provided variations in the 'cross-Squire' directions are small the analysis is similar to the two-dimensional case. The situation where the cross-Squire variations are not small has been examined in Gajjar \& Arebi (1995) for non-stationary vortices, and in Gajjar (1995) for the stationary cross-flow vortex. In both cases the analysis lead to a novel integro-differential equation for the vortex amplitude involving spanwise derivatives of the amplitude in the kernal function as in Wu (1993).

For low frequency waves, for certain wave angles, there exists two critical layers in the flow one located near the wall and the other in the main part of the boundary layer. The flow structure and analysis are therefore very similar to Gajjar (1994) with modifications for the additional critical layer which occurs in the three-dimensional case. Implicit in the present analysis is that there is no region of locally supersonic flow near the wall, in the terminology of Mack (1984). This places a certain restriction on the Mach number.

In section 2 below the problem is formulated for a compressible fluid satisfying the perfect gas law, and in sections 3 through 5 the analysis leading to the equations governing the evolution of the vortex is given. Finally we end with some discussion in section 6 .

\section{Formulation of the Instability Equations}

Consider coordinates $x^{\dagger}=\left(x^{\dagger}, y^{\dagger}, z^{\dagger}\right)$ such that $x^{\dagger}$ is directed along the wall, $y^{\dagger}$ is perpendicular to the wall and $z^{\dagger}$ is in the spanwise direction and such that the corresponding velocity components are given by $\mathbf{u}^{\dagger}=\left(u^{\dagger}, v^{\dagger}, w^{\dagger}\right)$, where a suffix $\dagger$ denotes a dimensional quantity. The Navier-Stokes equations are non-dimensionalised by taking $L$ to be a typical streamwise lengthscale and $U_{\infty}^{\dagger}$, a free stream velocity. Also a quantity $\phi_{\infty}^{\dagger}$ below denotes a local free stream value. The coordinates $\left(x^{\dagger}, y^{\dagger}, z^{\dagger}\right)$ are written as $(L x, L y, L z)$, the velocity $\left(u^{\dagger}, v^{\dagger}, w^{\dagger}\right)$ as $\left(U_{\infty}^{\dagger} u, U_{\infty}^{\dagger} v, U_{\infty}^{\dagger} w\right)$, the pressure $p^{\dagger}$ is scaled on $\rho^{\dagger} U_{\infty}^{\dagger^{2}} p$, the viscosity $\mu^{\dagger}$ is scaled on $\mu_{\infty}^{\dagger} \mu$, the bulk viscosity $\lambda^{\dagger}$ is scaled with respect to $\mu_{\infty}^{\dagger} \lambda$, the temperatute $T^{\dagger}$ is scaled with respect to $T_{\infty}^{\dagger} T$, the density $\rho^{\dagger}$ is scaled with $\rho_{\infty}^{\dagger} \rho$, and finally the conductivity $k^{\dagger}$ is scaled with $k_{\infty}^{\dagger} k$. The non-dimensional form of the equations are:

$$
\begin{gathered}
\frac{\partial \rho}{\partial t}+\frac{\partial}{\partial x_{j}}\left(\rho u_{j}\right)=0 \\
\frac{\partial u_{i}}{\partial t}+u_{j} \frac{\partial u_{i}}{\partial x_{j}}=-\frac{1}{\rho} \frac{\partial}{\partial x_{j}}\left(p \delta_{i j}\right)+\frac{1}{R}\left\{\frac{\partial}{\partial x_{j}}\left(\mu e_{i j}+\left(\lambda-\frac{2}{3} \mu\right) e_{k k}\right)\right\} \\
\rho\left[\frac{\partial p}{\partial t}+u_{i} \frac{\partial p}{\partial x_{i}}\right]=\Gamma p\left[\frac{\partial \rho}{\partial t}+u_{i} \frac{\partial \rho}{\partial x_{i}}\right]+\rho \frac{(\Gamma-1)}{R}\left[\frac{1}{2} \mu e_{i j} e_{i j}+\left(\lambda-\frac{2}{3} \mu\right)\left(\frac{\partial u_{i}}{\partial x_{i}}\right)^{2}\right] \\
+\frac{\rho}{R M_{\infty}^{2}} \frac{\partial}{\partial x_{i}}\left(\frac{\mu}{\operatorname{Pr}} \frac{\partial T}{\partial x_{i}}\right)
\end{gathered}
$$




$$
\begin{gathered}
\Gamma M_{\infty}^{2} p=\rho T, \\
e_{i j}=\left\{\frac{\partial u_{i}}{\partial x_{j}}+\frac{\partial u_{j}}{\partial x_{i}}\right\} .
\end{gathered}
$$

In the above equations the Reynolds number $R$, Mach number $M_{\infty}$, and the Prandtl number $P r$ are defined by

$$
R=\frac{U_{\infty}^{\dagger} L \rho_{\infty}^{\dagger}}{\mu_{\infty}^{\dagger}}, \quad M_{\infty}^{2}=\frac{U_{\infty}^{\dagger 2}}{\Gamma \mathcal{R} T_{\infty}}, \quad \operatorname{Pr}=\frac{c_{p} \mu_{\infty}^{\dagger}}{k_{\infty}^{\dagger}}
$$

and $c_{p}$ is the specific heat at constant pressure, $\Gamma$ is the ratio of specific heats, and $\mathcal{R}$ is the gas constant. The Reynolds number is assumed to be large and the Prandtl number to be constant.

The leading order three-dimensional basic boundary layer flow is taken to be of the form

$$
\begin{gathered}
\mathrm{U}_{B}=\left(U_{B}(x, Y, z), R^{-1 / 2} V_{B}(x, Y, z), W_{B}(x, Y, z)\right), \\
p=p_{B}(x, z), \quad T=T_{B}(x, Y, z), \quad \rho_{B}=\rho_{B}(x, Y, z),
\end{gathered}
$$

where $y=R^{-\frac{1}{2}} Y$ defines the boundary layer coordinate $Y$.

The analysis is simplified through the introduction of Squire coordinates $(\zeta, \bar{z})$, see Figure 1, along and normal to the direction of propagation of the wave, as follows

$$
\begin{gathered}
\frac{\partial}{\partial x} \rightarrow R^{\frac{1}{2}}\left(\alpha h \frac{\partial}{\partial \zeta}+\alpha h^{2} \frac{\partial}{\partial X}-\beta h \frac{\partial}{\partial \bar{z}}\right), \\
\frac{\partial}{\partial z} \rightarrow R^{\frac{1}{2}}\left(\beta h \frac{\partial}{\partial \zeta}+\beta h^{2} \frac{\partial}{\partial X}+\alpha h \frac{\partial}{\partial \bar{z}}\right), \\
\frac{\partial}{\partial t} \rightarrow R^{\frac{\partial}{2}} h^{2}(-\alpha c+h \sigma) \frac{\partial}{\partial \zeta},
\end{gathered}
$$

where the quantities $\zeta, \bar{z}$ and $X$ in (2.3) are defined by $\zeta=R^{\frac{1}{2}} h(\alpha x+\beta z-\alpha c h t)$, $\bar{z}=R^{\frac{1}{2}} h(\alpha z-\beta x)$ and $X=R^{\frac{1}{2}} h^{2}(\alpha x+\beta z)$. The wave is taken to grow on the slow $X$ scale. In equations (2.3) $\alpha$ and $\beta$ are defined by $\alpha=\cos \theta, \beta=\sin \theta$, where $\theta$ is the direction of propagation of the wave. Furthermore $c$ denotes the phase speed of the wave and $\sigma$ is a real quantity. The quantity $h$ is a scale factor $(h<<1)$ introduced such that we are considering long waves (based upon the Rayleigh scalings) and the growth rates are therefore $O\left(h^{2}\right)$.

The cross-flow velocity field is defined by $\bar{u}=\alpha u+\beta w, \bar{w}=-\beta u+\alpha w$ with respect to the chosen Squire coordinates. With respect to these coordinates the basic flow velocities are given by,

$$
\bar{U}_{B}=\alpha U_{B}+\beta W_{B}, \quad \bar{W}_{B}=-\beta U_{B}+\alpha W_{B},
$$


in the $\zeta$ and $\bar{z}$ directions respectively.

The stability analysis depends crucially on the properties of the basic flow near the wall and the upper critical level and it is assumed that

$$
\begin{aligned}
\bar{U}_{B} & \sim \lambda_{1} Y+\lambda_{2} Y^{2}+\ldots \text { as } Y \rightarrow 0+, \\
\rho_{B} & \sim R_{0}+R_{1} Y+\ldots \text { as } Y \rightarrow 0+, \\
T_{B} & \sim S_{0}+S_{1} Y+\ldots \text { as } Y \rightarrow 0+, \\
\bar{W}_{B} & \sim \delta_{1} Y+\delta_{2} Y^{2}+\ldots \text { as } Y \rightarrow 0+, \\
\bar{U}_{B} & \sim \lambda_{1}^{*}\left(Y-Y_{U}\right)+\lambda_{2}^{*}\left(Y-Y_{U}\right)^{2}+\ldots \text { as } Y \rightarrow Y_{U}, \\
\rho_{B} & \sim R_{0}^{*}+R_{1}^{*}\left(Y-Y_{U}\right)+\ldots \text { as } Y \rightarrow Y_{U}, \\
T_{B} & \sim S_{0}^{*}+S_{1}^{*}\left(Y-Y_{U}\right)+\ldots \text { as } Y \rightarrow Y_{U}, \\
\bar{W}_{B} & \sim \delta_{0}^{*}+\delta_{1}^{*}\left(Y-Y_{U}\right)+\ldots \text { as } Y \rightarrow Y_{U},
\end{aligned}
$$

where the upper critical level is defined by $\vec{U}_{B}=0$ at $Y=Y_{U}$. In addition at the outer edge of the boundary layer we require that

$$
\bar{U}_{B} \sim U_{\infty}, \quad \bar{W}_{B} \sim W_{\infty}, \quad \rho_{B} \sim \rho_{\infty}, \quad T_{B} \sim T_{\infty}, \quad \text { as } \quad Y \rightarrow \infty,
$$

and these outer edge values may depend on $x$ and $z$ in general.

A typical cross-flow velocity profile is of the form as shown in Figure 1 so that $\lambda_{1}$ and $\lambda_{1}^{*}$ are of opposite signs. Furthermore the combination $\lambda_{1} c$ is positive.

The main purpose of the analysis below is to determine the wavenumber and the linear and nonlinear growth rate of the vortex for a presribed mean flow and given frequency $\omega$. The wave-angle $\theta$ is taken to be a parameter and for a fixed value of $\theta$ the position $Y_{U}$ is also fixed.

\section{Stability analysis outside critical layers.}

We carry out a perturbation analysis by introducing small disturbances of size $\delta$ and considering expansions for the perturbed flow quantities. If we ignore the upper critical layer the bulk of the expansions below follow from those given in Gajjar (1994). The disturbance size $\delta$ will be fixed subsequently to be of $O\left(h^{3}\right)$ such that nonlinear effects first alter the linear growth rate. For clarity however, and since the flow structure outside the critical layer is largely dictated by linear dynamics, we work with $\delta$ for the time being. We first consider the main part of the boundary layer flow for which $Y=O(1)$ and expand the various flow quantities as

$$
\begin{gathered}
\bar{u}=\bar{U}_{B}+\delta\left(\bar{u}_{1}+h \bar{u}_{2}+\ldots\right), \\
v=\delta\left(h \bar{v}_{1}+h^{2} \bar{v}_{2}+\ldots\right),
\end{gathered}
$$




$$
\begin{gathered}
\bar{w}=\bar{W}_{B}+\delta\left(\tilde{w}_{1}+h \tilde{w}_{2}+\ldots\right), \\
p=p_{B}+\delta\left(h \bar{p}_{1}+h^{2} \bar{p}_{2}+\ldots\right), \\
\rho=\rho_{B}+\delta\left(\bar{\rho}_{1}+h \bar{\rho}_{2}+\ldots\right),
\end{gathered}
$$

(and the perturbed quantities are taken to be independent of $\bar{z}$ to this order).

These expansions (3.1) are substituted into the governing equations (2.1) yielding the following solutions at leading order

$$
\begin{gathered}
\bar{u}_{1}=\bar{A}_{0} \bar{U}_{B_{Y}}, \quad \bar{v}_{1}=-\tilde{A}_{0_{\zeta}} \bar{U}_{B}, \\
\bar{p}_{1}=\bar{P}_{1}, \quad \bar{\rho}_{1}=\bar{A}_{0} \rho_{B_{Y}}, \quad \bar{w}_{1}=\bar{A}_{0 \varsigma} \bar{W}_{B_{Y}}
\end{gathered}
$$

where $\tilde{A}_{0}$ is given by $\tilde{A}_{0}=\tilde{A}(X) \exp (i \gamma \zeta)+$ c.c. and $\tilde{P}_{1}=\bar{P}_{11}(X) \exp (i \gamma \zeta)+$ c.c. and $\gamma$ is the wavenumber of the cross-flow vortex.

At next order the analysis yields

$$
\begin{gathered}
\bar{p}_{2}=\tilde{P}_{2}-\int_{0}^{Y} \bar{U}_{B \rho_{B}} \bar{v}_{1_{\zeta}} d Y, \\
\bar{v}_{2}=\bar{U}_{B} \int_{Y_{0}}^{Y} \frac{\tilde{p}_{1_{\zeta}}}{\rho_{B} \bar{U}_{B}^{2}} d Y+\alpha c \bar{A}_{0_{\zeta}}-\bar{A}_{1}^{ \pm} \bar{U}_{B}-\frac{\bar{U}_{B} \bar{p}_{1_{\zeta}}}{\Gamma p_{B}} Y . \\
\bar{w}_{2}=-\frac{\bar{A}_{0_{X}} \bar{W}_{B_{Y}}}{i \gamma}-\bar{W}_{B_{Y}}\left(\int_{Y_{0}}^{Y} \frac{\bar{p}_{1}}{\rho_{B} \bar{U}_{B}^{2}} d Y-\frac{\tilde{A}_{1}^{ \pm}}{i \gamma}-\frac{\bar{p}_{1}}{\Gamma p_{B}} Y\right) .
\end{gathered}
$$

The constant $Y_{0}$ is chosen to be such that $Y_{0}>Y_{U}$. It can be seen that the integrals in equation (3.3bc) are singular at the locations where $\bar{U}_{B}=0$ and this occurs at $Y=0$ and $Y=Y_{U}$ (see Figure 2). Additional expansions must be introduced in order to obtain valid expressions for the flow quantities near $Y=0$ and $Y=Y_{U}$. In the neighbourhood of the lower critical layer situated near the wall we introduce regions $I \pm$ of thickness $O(h)$ and in the vicinity of $Y_{U}$ (the upper critical level situated in the main part of the boundary layer flow) regions $I V \pm$ are introduced (again of thickness $O(h)$ ). Hence the $Y=O(1)$ region is divided into two regions $I I \pm$ due to the presence of the upper critical layer $Y_{U}$ in the main part of the boundary layer, see Figure 2. Moreover we define the unknown functions $\tilde{A}_{1}^{ \pm}$in equation $(3.3 b)$ such that $\bar{A}_{1}^{-}$relates to region $I I-$ and $\tilde{A}_{1}^{+}$relates to region $I I+$ where $\tilde{A}_{1}^{ \pm}$(and $\tilde{P}_{2}$ ) are functions of $\zeta$ and $X$.

Considering first the regions $I_{ \pm}$of thickness $O(h)$ close to the wall we introduce a coordinate $\bar{Y}$ such that $Y=h \bar{Y}$ and the above solutions therefore imply

$$
\bar{u}=h \lambda_{1} \bar{Y}+h^{2} \lambda_{2} \bar{Y}^{2}+\ldots+\delta\left(\bar{u}_{1}+h \bar{u}_{2}+\ldots\right),
$$




$$
\begin{gathered}
v=\delta\left(h^{2} \bar{v}_{1}+h^{3} \bar{v}_{2}+\ldots\right), \\
p=p_{B}+\delta\left(h \bar{p}_{1}+h^{2} \bar{p}_{2}+\ldots\right), \\
\rho=R_{0}+h R_{1} \bar{Y}+h^{2} R_{2} \bar{Y}^{2}+\delta\left(\bar{\rho}_{1}+h \bar{\rho}_{2}+\ldots\right), \\
\bar{w}=\delta_{0}+h \delta_{1} \bar{Y}+\ldots+\delta\left(\bar{w}_{1}+h \bar{w}_{2}+\ldots\right) .
\end{gathered}
$$

Substituting into the governing equations we obtain at leading order the solutions

$$
\begin{gathered}
\bar{u}_{1}=\lambda_{1} \tilde{A}_{0}, \quad \bar{v}_{1}=\frac{-\bar{p}_{1_{\zeta}}}{\lambda_{1} R_{0}}-\bar{A}_{0_{\zeta}}\left(\lambda_{1} \bar{Y}-\alpha c\right), \\
\bar{p}_{1}=\bar{P}_{1} \exp (i \gamma \zeta), \quad \bar{\rho}_{1_{\zeta}}=\frac{-\bar{v}_{1} R_{1}}{\left(\lambda_{1} \bar{Y}-\alpha c\right)}, \quad \bar{w}_{1 \zeta}=-\frac{\delta_{1} \bar{v}_{1}}{\left(\lambda_{1} \bar{Y}-\alpha c\right)} .
\end{gathered}
$$

We note the density function has a pole singularity at $Y_{L}=\alpha c / \lambda_{1}$ (as indeed does the $\bar{w}_{1}$ fluctuation). The requirement that $\bar{v}_{1} \rightarrow 0$ as $\bar{Y} \rightarrow 0$ gives

$$
\bar{P}_{1}=\bar{A} \alpha c \lambda_{1} R_{0} .
$$

At the next order we find

$$
\begin{gathered}
\bar{v}_{2}=\left(-\sigma \bar{A}_{0_{\zeta}}-\alpha c \tilde{A}_{0_{x}}\right)-\frac{\lambda_{2} \bar{A}_{0_{\zeta}}\left(\bar{\eta}^{2}-\alpha^{2} c^{2}\right)}{\lambda_{1}^{2}}+\frac{1}{R_{0} \lambda_{1}}\left(R_{1} \alpha^{2} c^{2} \tilde{A}_{0_{\zeta}}-\bar{p}_{2_{\zeta}}-\bar{p}_{1_{x}}\right)- \\
\frac{\alpha c \bar{A}_{0_{\zeta}}}{\lambda_{1}}\left\{\frac{R_{1}}{R_{0}}+\frac{2 \lambda_{2}}{\lambda_{1}}\right\}\left(\ln |\bar{\eta}|+\phi_{1}^{ \pm}\right) \bar{\eta} \\
\bar{p}_{2}=\bar{p}_{2}(\zeta, X)
\end{gathered}
$$

where $\phi_{1}^{ \pm}$are terms arising from the continuation across the lower critical layer at $\bar{\eta}=0$, (where $\bar{\eta}=\lambda_{1} \bar{Y}-\alpha c$ ) and $\bar{p}_{2}$ is an unknown function of $\zeta$ and $X$.

We now consider the Regions $I V \pm$ near to the upper critical layer situated at $Y=Y_{U}$ and write $Y=Y_{U}+h Y^{*}$.

From the solutions in region $I I$ the flow quantities are expanded as

$$
\begin{gathered}
\bar{u}=h \lambda_{1}^{*} Y^{*}+h^{2} \lambda_{2}^{*} Y^{* 2}+\delta\left(u_{1}^{*}+h u_{2}^{*}+\ldots\right), \\
v=\delta\left(h^{2} v_{1}^{*}+h^{3} v_{2}^{*}+\ldots\right), \\
p=p_{B}+\delta\left(h p_{1}^{*}+h^{2} p_{2}^{*}+\ldots\right), \\
\rho=R_{0}^{*}+h R_{1}^{*} Y^{*}+h^{2} R_{2}^{*} Y^{* 2}+\delta\left(\rho_{1}^{*}+h \rho_{2}^{*}+\ldots\right),
\end{gathered}
$$




$$
\bar{w}=\delta_{0}^{*}+h \delta_{1}^{*} Y^{*}+\ldots+\delta\left(w_{1}^{*}+h w_{2}^{*}+\ldots\right),
$$

and substituting into the governing equations (2.1) yields at leading order

$$
\begin{gathered}
v_{1}^{*}=-\frac{p_{1_{\zeta}}^{*}}{\lambda_{1}^{*} R_{0}^{*}}-\tilde{A}_{0_{\zeta}}\left(\lambda_{1}^{*} Y^{*}-\alpha c\right), \\
p_{1}^{*}=\left(P_{1}^{*} \exp (i \gamma \zeta)+c . c\right) .
\end{gathered}
$$

At next order

$$
\begin{gathered}
v_{2}^{*}=-\left(\sigma \tilde{A}_{0_{\zeta}}+\alpha c \bar{A}_{0_{x}}\right)+\frac{1}{\lambda_{1}^{*} R_{0}^{*}}\left\{\frac{2 \lambda_{2}^{*} \alpha c p_{1_{\zeta}}^{*}}{\lambda_{1}^{* 2}}+\frac{R_{1}^{*} \alpha c p_{1_{\zeta}}^{*}}{\lambda_{1}^{*} R_{0}^{*}}-p_{2_{\zeta}}^{*}-p_{1_{X}}^{*}\right\}- \\
\frac{\lambda_{2}^{*} \bar{A}_{0_{\zeta}}}{\lambda_{1}^{* 2}} \bar{\eta}_{1}^{2}-p_{1 \zeta}^{*}\left\{\frac{2 \lambda_{2}^{*}}{\lambda_{1}^{* 3} R_{0}^{*}}+\frac{R_{1}^{*}}{R_{0}^{* 2} \lambda_{1}^{* 2}}\right\} \bar{\eta}_{1}\left(\ln \left(\left|\bar{\eta}_{1}\right|+\phi_{2}^{ \pm}\right),\right. \\
p_{2}^{*}=p_{2}^{*}(\zeta, X),
\end{gathered}
$$

where $\bar{\eta}_{1}=\lambda_{1}^{*} Y^{*}-\alpha c$ and the $\phi_{2}^{ \pm}$terms are from the continuation across the upper critical layer at $\bar{\eta}_{1}=0, P_{1}^{*}$ is an unknown function of $X$ and $p_{2}^{*}$ is an unknown function of $\zeta$ and $X$.

The boundary layer flow must also match with the core flow outside of the boundary layer region hence we consider a far field region (region $I I I$ Figure 2) where $Y=\hat{Y} / h$ and expand in the following manner

$$
\begin{gathered}
\bar{u}=U_{\infty}+\delta\left(h \hat{u}_{1}+h^{2} \hat{u}_{2}+\ldots\right), \\
v=\delta\left(h \hat{v}_{1}+h^{2} \hat{v}_{2}+\ldots\right) \\
\rho=\rho_{\infty}+\delta\left(h \hat{\rho}_{1}+\ldots\right) \\
p=p_{B}+\delta\left(h \hat{p}_{1}+h^{2} \hat{p}_{2}+\ldots\right) .
\end{gathered}
$$

Substitution into the governing equations yields at leading order the Prandtl-Glauert equation for $\hat{p}_{1}$ with solution

$$
\hat{p}_{1}=\left(\bar{P}_{11} \exp (-\gamma \Omega \hat{Y}) \exp (i \gamma \zeta)+c . c\right),
$$

where

$$
\Omega^{2}=1-\frac{U_{\infty}^{2} M_{\infty}^{2}}{T_{\infty}}
$$

and

$$
\hat{v}_{1}=v_{10} \exp (-\gamma \Omega \hat{Y}) \exp (i \gamma \zeta)
$$




$$
v_{10}=-\frac{i \bar{P}_{11} \Omega}{\rho_{\infty} U_{\infty}} .
$$

Real quantities are assumed and $\bar{P}_{11}$ is an unknown function of $X$. At the next order the $e^{i \gamma \zeta}$ component of $\hat{p}_{2}$, say $\hat{p}_{21}$, satisfies

$$
\hat{p}_{21_{\hat{y} \dot{y}}}+\Omega^{2} \hat{p}_{21_{\zeta \zeta}}=-2 \alpha c U_{\infty} \frac{\rho_{\infty}}{\Gamma p_{B}} \hat{p}_{1_{\zeta \zeta}}-\Omega^{2} \hat{p}_{1_{x \zeta}}-i \gamma \Omega^{2} \hat{p}_{1_{x}} .
$$

The solution is given by

$$
\hat{p}_{21}=P_{21} \exp (-\gamma \Omega \hat{Y}) \exp (i \gamma \zeta)
$$

where

$$
P_{21}=\bar{P}_{21}-\frac{\alpha c\left(1-\Omega^{2}\right) \gamma \bar{P}_{11} \hat{Y}}{\Omega U_{\infty}}+i \Omega \bar{P}_{11_{x}} \hat{Y}
$$

and $\bar{P}_{21}(X)$ is a constant of integration. We note that higher harmonics are also present in $\hat{p}_{2}$. The $e^{i \gamma \zeta}$ component of the second order normal velocity disturbance $\hat{v}_{2}$ can then be determined such that

$$
\hat{v}_{2}=v_{20} \exp (-\gamma \Omega \hat{Y}) \exp (i \gamma \zeta)
$$

where

$$
i \rho_{\infty} \gamma U_{\infty} v_{20}=\frac{\alpha c \gamma}{U_{\infty} \Omega}+\gamma \Omega \bar{P}_{21}
$$

\section{Derivation of the Amplitude Equation}

The amplitude equation governing the evolution of the mode is obtained from matching the expressions obtained for the pressure function and the normal velocity across the various regions of the flow.

It is convenient to denote the $e^{i \gamma \zeta}$ components of $\tilde{P}_{2}, \bar{p}_{2}, \bar{A}_{1}^{ \pm}, \bar{A}_{0} \phi_{1}^{ \pm}, \bar{A}_{0} \phi_{2}^{ \pm}$by $\bar{P}_{21}, \bar{p}_{21}$ $\bar{A}_{11}^{ \pm}, \bar{A} \phi_{11}^{ \pm}, \bar{A} \phi_{21}^{ \pm}$, respectively. The matching of pressure components across regions $I I+$ and $I I I$ (equations (3.2c), (3.3a), (3.12) and (3.15)) yields at leading order

$$
\bar{P}_{11}=\bar{P}_{11}
$$

and at next order matching gives

$$
\bar{P}_{21}=\bar{P}_{21}-\bar{A} \gamma^{2} I_{1}
$$

where

$$
I_{1}=\int_{0}^{\infty}\left(\rho_{B} \bar{U}_{B}^{2}-\rho_{\infty} U_{\infty}^{2}\right) d Y .
$$

In addition from the matching of the leading order normal velocity components in regions $I I^{+}$and $I I I$ we obtain

$$
\Omega \bar{P}_{11}=\bar{A} U_{\infty}^{2} \gamma \rho_{\infty}
$$


Matching the pressures between $\mathrm{I}^{+}$and $\mathrm{II}^{-}$shows that

$$
\tilde{P}_{11}=\bar{P}_{1}
$$

and hence combining (3.6), (4.4) gives the dispersion relation

$$
\gamma \rho_{\infty} U_{\infty}^{2}=\lambda_{1} \Omega R_{0} \alpha c .
$$

Next matching the normal velocity components in regions ${ }^{+} I^{+}$and $I I I$ at higher order gives using (3.16b),

$$
-\bar{A}_{11}^{+} U_{\infty}+D_{1} \bar{A}=\frac{\gamma \Omega}{i \gamma U_{\infty} \rho_{\infty}} \tilde{P}_{21}
$$

where

$$
D_{1}=\left[i \gamma U_{\infty} I_{2}^{\infty}-\frac{i \gamma Y_{0}}{\rho_{\infty} U_{\infty}}+\frac{i \alpha c}{\Omega U_{\infty}^{2}}\right]\left(\frac{\bar{P}_{11}}{\bar{A}}\right)+i \gamma \alpha c-\frac{i \gamma^{2} \Omega}{U_{\infty}} I_{1},
$$

and $I_{2}^{\infty}$ is a finite quantity given by

$$
I_{2}^{\infty}=\int_{Y_{0}}^{\infty}\left\{\frac{1}{\rho_{B} \bar{U}_{B}^{2}}-\frac{1}{\rho_{\infty} U_{\infty}^{2}}\right\} d Y .
$$

Matching the pressure terms (equations (3.2c), (3.3a), (3.5c) and (3.7b)) across regions $I+$ and $I I$ - gives

$$
\tilde{P}_{11}=\bar{P}_{11}
$$

and at next order

$$
\bar{P}_{21}=\bar{p}_{21},
$$

Matching the normal velocity components across these two regions (equations (3.2a), $(3.3 b),(3.5 b)$ and $(3.7 a))$ yields

$$
-i \gamma \alpha c \tilde{A}\left\{\frac{2 \lambda_{2}}{\lambda_{1}}+\frac{R_{1}}{R_{0}}\right\} \phi_{11}^{+}=-\tilde{A}_{11}^{-} \lambda_{1}+D_{2} \tilde{A}
$$

where

$$
\begin{aligned}
D_{2}=-\frac{2 i \gamma \lambda_{2} \alpha c}{\lambda_{1}} & +i \gamma \lambda_{1}\left(\frac{\bar{P}_{11}}{\bar{A}}\right) I_{2}^{(0)}+i \gamma\left(\frac{\bar{P}_{11}}{\tilde{A}}\right) \lambda_{1}\left[\frac{1}{R_{0}^{*} \lambda_{1}^{* 2}}\left(\frac{1}{Y_{0}}+\frac{1}{Y_{0}-Y_{u}}\right)\right. \\
& \left.+\frac{1}{R_{0} \lambda_{1}^{2} Y_{0}}+\left(\frac{R_{1}^{*}}{R_{0}^{*}}+\frac{2 \lambda_{2}^{*}}{\lambda_{1}^{*}}\right) \frac{1}{R_{0}^{*} \lambda_{1}^{* 2}} \ln \left|\frac{Y_{u}}{Y_{0}-Y_{u}}\right|-\frac{1}{R_{0} \lambda_{1}^{2}} \ln \left|Y_{0}\right|\left(\frac{R_{1}}{R_{0}}+\frac{2 \lambda_{2}}{\lambda_{1}}\right)\right]
\end{aligned}
$$

and

$$
\begin{aligned}
I_{2}^{(0)} & =\int_{Y_{0}}^{0}\left[\frac{1}{\rho_{B} U_{B}^{2}}-\frac{1}{R_{0}^{*} \lambda_{1}^{* 2}\left(Y-Y_{u}\right)^{2}}-\frac{1}{R_{0} \lambda_{1}^{2} Y^{2}}\right. \\
& \left.+\frac{1}{R_{0}^{*} \lambda_{1}^{* 2}\left(Y-Y_{u}\right)}\left(\frac{R_{1}^{*}}{R_{0}^{*}}+\frac{2 \lambda_{2}^{*}}{\lambda_{1}^{*}}\right)+\frac{1}{R_{0} \lambda_{1}^{2} Y}\left(\frac{R_{1}}{R_{0}}+\frac{2 \lambda_{2}}{\lambda_{1}}\right)\right] d Y .
\end{aligned}
$$


Further on applying the boundary condition at the wall (namely that the normal velocity component at the wall is zero) (3.7a) implies that

$$
-i \sigma \gamma \tilde{A}-2 \alpha c \tilde{A}_{X}-\frac{i \gamma \tilde{P}_{21}}{R_{0} \lambda_{1}}+\frac{i \gamma \alpha^{2} c^{2}}{\lambda_{1}}\left\{\frac{2 \lambda_{2}}{\lambda_{1}}+\frac{R_{1}}{R_{0}}\right\}\left(\phi_{11}^{-}+\ln |\alpha c|\right) \bar{A}+\frac{i \gamma R_{1} \alpha^{2} c^{2}}{R_{0} \lambda_{1}} \tilde{A}=0 .
$$

We now consider matching the normal velocity components (equations $(3.2 a),(3.3 b)$, (3.9a) and (3.10a)) across regions $I I$ and $I V$. This shows that

$$
\tilde{A}_{11}^{+}-\bar{A}_{11}^{-}=\frac{i \gamma \tilde{P}_{11}}{\lambda_{1}^{* 2} R_{0}^{*}}\left\{\frac{2 \lambda_{2}^{*}}{\lambda_{1}^{*}}+\frac{R_{1}^{*}}{R_{0}^{*}}\right\}\left(\phi_{21}^{+}-\phi_{21}^{-}\right) .
$$

Then on elimination of $\bar{A}_{11}^{-}$and $\tilde{A}_{11}^{+}$between (4.6), (4.8c), (4.8d) and (4.9) leads to the amplitude equation

$$
\begin{aligned}
D_{3} \bar{A}-2 \alpha c \bar{A}_{X}-\frac{i \gamma \bar{A} \alpha^{2} c^{2}}{\lambda_{1}} & \left\{\frac{2 \lambda_{2}}{\lambda_{1}}+\frac{R_{1}}{R_{0}}\right\}\left(\phi_{11}^{+}-\phi_{11}^{-}\right)= \\
& +\frac{i \gamma \alpha^{2} c^{2} \lambda_{1} \tilde{A}}{\lambda_{1}^{* 2}}\left\{\frac{2 \lambda_{2}^{*}}{\lambda_{1}^{*}}+\frac{R_{1}^{*}}{R_{0}^{*}}\right\}\left(\phi_{21}^{+}-\phi_{21}^{-}\right) \frac{R_{0}}{R_{0}^{*}}
\end{aligned}
$$

where $D_{3}$ is a purely imaginary constant defined by

$$
D_{3}=\frac{\gamma U_{\infty} \rho_{\infty}}{R_{0} \lambda_{1} \Omega} D_{1}-i \gamma \sigma+\frac{i \gamma R_{1}(\alpha c)^{2}}{R_{0} \lambda_{1}}+\frac{i \gamma(\alpha c)^{2}}{\lambda_{1}}\left(\frac{R_{1}}{R_{0}}+\frac{2 \lambda_{2}}{\lambda_{1}}\right) \ln |\alpha c|-\frac{\gamma U_{\infty}^{2} \rho_{\infty}}{R_{0} \lambda_{1}^{2} \Omega} D_{2} .
$$

Equation (4.10) shows that the amplitude of the cross-flow vortex is dependent on the jumps $\left(\phi_{11}^{+}-\phi_{11}^{-}\right)$and $\left(\phi_{21}^{+}-\phi_{21}^{-}\right)$across the lower and upper critical layers. We consider next the details of the critical layers.

\section{Critical layer Analysis}

By increasing the size of $\delta$ it is found that when $\delta$ is of order $O\left(h^{3}\right)$ nonlinearity significantly alters the linear growth rate. This is the same as in Gajjar (1994) where some further justification and a balancing argument may be found. It is interesting to note that in this long wavelength limit nonlinearity gives rise to an unsteady nonlinear partial differential equation system governing the evolution of the disturbances, in contrast to shorter waves where nonlinearity leads to a single Hickernell (1984) type of integrodifferential equation.

We first consider the lower critical layer region (of thickness $O\left(h^{2}\right)$ ) and write $Y=$ $h \frac{\alpha c}{\lambda_{1}}+h^{2} \hat{\eta}$. The flow quantities are expanded in the following manner

$$
\bar{u}=h \alpha c+h^{2}\left(\lambda_{1} \hat{\eta}+\frac{\lambda_{2} \alpha^{2} c^{2}}{\lambda_{1}^{2}}\right)+h^{3} \bar{U}_{1}^{*}+h^{4} \bar{U}_{2}^{*}+\ldots
$$




$$
\begin{gathered}
v=h^{5} \bar{V}_{0}^{*}+h^{6} \bar{V}_{1}^{*}+h^{7} \bar{V}_{2}^{*}+\ldots, \\
p=p_{B}+h^{4} \bar{P}_{1}^{*}+h^{5} \bar{P}_{2}^{*}+\ldots \\
\rho=R_{0}+\frac{h R_{1} \alpha c}{\lambda_{1}}+h^{2} \Pi_{1}+\ldots \\
\bar{w}=h \frac{\delta_{1} \alpha c \hat{\eta}}{\lambda_{1}}+h^{2} \bar{W}_{1}^{*}+\ldots
\end{gathered}
$$

On substitution into the governing equations (2.1) we obtain (at the first non-trivial order)

$$
\begin{gathered}
L\left(\Pi_{1}\right)=0, \\
L\left(q_{L}\right)=\frac{\Pi_{1_{i}} \bar{P}_{l_{c}}^{*}}{R_{0}^{2}},
\end{gathered}
$$

where $L$ is the operator defined by

$$
L=\left\{\sigma+\lambda_{1} \hat{\eta}+\frac{\lambda_{2} \alpha^{2} c^{2}}{\lambda_{1}^{2}}\right\} \frac{\partial}{\partial \zeta}+\alpha c \frac{\partial}{\partial X}+\bar{V}_{0}^{*} \frac{\partial}{\partial \hat{\eta}}
$$

and

$$
\bar{V}_{0}^{*}=-\frac{\bar{P}_{1_{\zeta}}^{*}}{R_{0} \lambda_{1}}, \quad q_{L}=\bar{U}_{2 \hat{\eta}^{*}}^{*}
$$

The upper critical layer, at $Y_{U}$, is considered next and we write $Y=Y_{U}+h \frac{\alpha c}{\lambda_{i}^{i}}+h^{2} \hat{\eta}_{1}$ and the flow quantities there expand as

$$
\begin{gathered}
\bar{u}=h \alpha c+h^{2}\left\{\lambda_{1}^{*} \hat{\eta}_{1}+\frac{\lambda_{2}^{*} \alpha^{2} c^{2}}{\lambda_{*}^{2}}\right\}+h^{3} \hat{U}_{1}^{*}+h^{4} \hat{U}_{2}^{*}+\ldots, \\
v=h^{5} \hat{V}_{0}^{*}+h^{6} \hat{V}_{1}^{*}+h^{7} \hat{V}_{2}^{*}+\ldots, \\
p=p_{B}+h^{4} \hat{P}_{1}^{*}+h^{5} \hat{P}_{2}^{*}+\ldots, \\
\rho=R_{0}^{*}+\frac{h R_{1}^{*} \alpha c}{\lambda_{1}^{*}}+h^{2} \Phi_{1}+\ldots
\end{gathered}
$$

Substitution into the compressible Navier-Stokes equations yields

$$
\begin{gathered}
L_{1}\left(\Phi_{1}\right)=0, \\
L_{1}\left(q_{U}\right)=\frac{\Phi_{1_{i_{1}}} \hat{P}_{1_{\zeta}}^{*}}{R_{0}^{* 2}}
\end{gathered}
$$


where $L_{1}$ is the operator given by

$$
L_{1}=\left\{\sigma+\lambda_{1}^{*} \hat{\eta}_{1}+\frac{\lambda_{2}^{*} \alpha^{2} c^{2}}{\lambda_{1}^{* 2}}\right\} \frac{\partial}{\partial \zeta}+\alpha c \frac{\partial}{\partial X}+\hat{V}_{0}^{*} \frac{\partial}{\partial \hat{\eta}_{1}},
$$

and

$$
\hat{V}_{0}^{*}=-\frac{\hat{P}_{1_{\zeta}}^{*}}{\lambda_{1}^{*} R_{0}^{*}}, \quad q_{U}=\hat{U}_{2 \hat{\eta}_{1}}^{*}
$$

The solutions in the lower critical layer region must be matched with the solutions in regions $I^{ \pm}$(of thickness $O(h)$ ) just outside of the critical layer so as to determine the behaviour of $Q_{L}$ and $\Pi_{L}$ as $\hat{\eta} \rightarrow \pm \infty$. Matching using (3.5) and (3.7) yields

$$
q_{L} \sim 2 \lambda_{2} \hat{\eta}+\frac{2 \alpha c}{\hat{\eta}}\left\{\frac{2 \lambda_{2}}{\lambda_{1}}+\frac{R_{1}}{R_{0}}\right\} \operatorname{Re}(\bar{A} \exp (i \gamma \zeta))+\ldots \quad \text { as } \quad \hat{\eta} \rightarrow \pm \infty,
$$

and

$$
\Pi_{1} \sim R_{1} \hat{\eta}+\frac{2 R_{1} \alpha c}{\lambda_{1} \hat{\eta}} \operatorname{Re}(\bar{A} \exp (i \gamma \zeta))+\ldots \quad \text { as } \quad \hat{\eta} \rightarrow \pm \infty .
$$

Similarly from matching the upper critical layer with regions $I V^{ \pm}$, equations (3.9) and (3.10) yields,

$$
q_{U} \sim 2 \lambda_{2}^{*} \hat{\eta}_{1}+\frac{2 \alpha c \lambda_{1} R_{0}}{\hat{\eta}_{1} \lambda_{1}^{*} R_{0}^{*}}\left\{\frac{2 \lambda_{2}^{*}}{\lambda_{1}^{*}}+\frac{R_{1}^{*}}{R_{0}^{*}}\right\} \operatorname{Re}(\tilde{A} \exp (i \gamma \zeta))+\ldots
$$

and

$$
\Phi_{1} \sim R_{1}^{*} \hat{\eta}_{1}+\frac{2 R_{1}^{*} \alpha c R_{0} \lambda_{1}}{\lambda_{1}^{* 2} \hat{\eta}_{1} R_{0}^{*}} \operatorname{Re}(\bar{A} \exp (\dot{i} \gamma \zeta))+\ldots
$$

as

$$
\hat{\eta}_{1} \rightarrow \pm \infty
$$

The solutions of (5.2), (5.3), (5.6) and (5.7) together with the amplitude equation, (4.10), govern the evolution of the vortex. We may rewrite the jump conditions in the form

$$
\int_{0}^{\frac{2 \pi}{\gamma}} \int_{-\infty}^{* \infty} q_{L} \exp (-i \gamma \zeta) d \hat{\eta} d \zeta=\frac{2 \pi \alpha c}{\gamma}\left\{\frac{2 \lambda_{2}}{\lambda_{1}}+\frac{R_{1}}{R_{0}}\right\} \bar{A}\left(\phi_{1}^{+}-\phi_{1}^{-}\right),
$$

and

$$
\int_{0}^{\frac{2 \pi}{\gamma}} \int_{-\infty}^{* \infty} q U \exp (-i \gamma \zeta) d \hat{\eta}_{1} d \zeta=\frac{2 \pi \alpha c \lambda_{1} R_{0}}{\gamma R_{0}^{*} \lambda_{1}^{*}}\left\{\frac{2 \lambda_{2}^{*}}{\lambda_{1}^{*}}+\frac{R_{1}^{*}}{R_{0}^{*}}\right\} \tilde{A}\left(\phi_{2}^{+}-\phi_{2}^{-}\right),
$$

with $\int^{*}$ denoting a finite part integral. This enables us to rewrite the amplitude equation as 


$$
\begin{gathered}
D_{3} \bar{A}-2 \alpha c \bar{A}_{X}-\frac{i \gamma^{2} \alpha c}{2 \pi \lambda_{1}} \int_{0}^{\frac{2 \pi}{\gamma}} \int_{-\infty}^{* \infty} q_{L} \exp (-i \gamma \zeta) d \hat{\eta} d \zeta= \\
\frac{i \gamma^{2} \alpha c}{2 \pi \lambda_{1}^{*}} \int_{0}^{\frac{2 \pi}{\gamma}} \int_{-\infty}^{* \infty} q_{U} \exp (-i \gamma \zeta) d \hat{\eta}_{1} d \zeta
\end{gathered}
$$

where the $D_{3}$ term gives a correction to the wavenumber.

A more standard form of the equations is obtained by re-normalising using the following scalings:

$$
\begin{gathered}
\lambda_{1} \hat{\eta}+\frac{\lambda_{2} \alpha^{2} c^{2}}{\lambda_{1}}+\sigma=\lambda_{1} d_{1}^{*} \hat{Z}, \\
\lambda_{1}^{*} \hat{\eta}_{1}+\frac{\lambda_{2}^{*} \alpha^{2} c^{2}}{\lambda_{1}^{*}}+\sigma=\lambda_{1}^{*} d_{4}^{*} \hat{Z}_{1}, \\
\Pi_{1}=R_{1} d_{1}^{*} \Pi, \quad \Phi_{1}=R_{1}^{*} d_{4}^{*} \Phi, \\
X=\frac{\alpha c}{\lambda_{1} \gamma d_{1}^{*}} \hat{X}-\hat{X}_{0}, \quad \gamma \zeta=\zeta^{*}+\zeta_{0}, \\
\tilde{A}=d_{0}^{*} A \exp \left(-i \zeta_{0}\right), \\
q_{L}=d_{2}^{*} Q_{L}, \quad q_{U}=d_{3}^{*} Q_{U},
\end{gathered}
$$

(where $\hat{X}_{0}$ and $\zeta_{0}$ are chosen so as to match with upstream conditions). The equations (5.3), (5.2), (5.7) and (5.6) respectively are rescaled as

$$
\begin{gathered}
\frac{\partial Q_{L}}{\partial \hat{X}}+\hat{Z} \frac{\partial Q_{L}}{\partial \zeta^{*}}-\operatorname{Re}\left(i A \exp \left(i \zeta^{*}\right)\right) \frac{\partial Q_{L}}{\partial \hat{Z}}=J \operatorname{Re}\left(i A \exp \left(i \zeta^{*}\right)\right) \frac{\partial \Pi}{\partial \hat{Z}} \\
\frac{\partial \Pi}{\partial \hat{X}}+\hat{Z} \frac{\partial \Pi}{\partial \zeta^{*}}-\operatorname{Re}\left(i A \exp \left(i \zeta^{*}\right)\right) \frac{\partial \Pi}{\partial \hat{Z}}=0 \\
\Delta \frac{\partial Q_{U}}{\partial \hat{X}}+\hat{Z}_{1} \frac{\partial Q_{U}}{\partial \zeta^{*}}-\operatorname{Re}\left(i A \exp \left(i \zeta^{*}\right)\right) \frac{\partial Q_{U}}{\partial \hat{Z}_{1}}=J_{1} \operatorname{Re}\left(i A \exp \left(i \zeta^{*}\right)\right) \frac{\partial \Phi}{\partial \hat{Z}_{1}} \\
\Delta \frac{\partial \Phi}{\partial \hat{X}}+\hat{Z}_{1} \frac{\partial \Phi}{\partial \zeta^{*}}-\operatorname{Re}\left(i A \exp \left(i \zeta^{*}\right)\right) \frac{\partial \Phi}{\partial \hat{Z}_{1}}=0
\end{gathered}
$$

and the amplitude equation (5.14) is transformed to

$$
\begin{aligned}
A_{\hat{X}}=-\frac{i \hat{E}_{1}}{\pi} \int_{0}^{2 \pi} \int_{-\infty}^{\infty} Q_{L} \exp \left(-i \zeta^{*}\right) d \zeta^{*} d \hat{Z}- \\
\frac{i \hat{E}_{2}}{\pi} \int_{0}^{2 \pi} \int_{-\infty}^{\infty} Q_{U} \exp \left(-i \zeta^{*}\right) d \zeta^{*} d \hat{Z}_{1}+\bar{D}_{3} A
\end{aligned}
$$


where

$$
J=\frac{\frac{R_{1}}{R_{0}}}{\left\{\frac{2 \lambda_{2}}{\lambda_{1}}+\frac{R_{1}}{R_{0}}\right\}}, \quad J_{1}=\frac{\frac{R_{i}^{*}}{R_{0}^{*}}}{\left\{\frac{2 \lambda_{i}^{*}}{\lambda_{i}^{*}}+\frac{R_{i}^{*}}{R_{0}^{*}}\right\}} .
$$

The constants $d_{i}^{*}, \Delta, \hat{E}_{1}, \hat{E}_{2}$ are given by

$$
\begin{gathered}
d_{1}^{*}=\frac{\alpha^{2} c^{2}}{2 \lambda_{1}^{2}}, \quad D_{3}=2 \lambda_{1} \gamma d_{1}^{*} \bar{D}_{3} \\
d_{0}^{*}=\frac{\lambda_{1} d_{1}^{* 2}}{2 \alpha c}, \quad(1-J) d_{2}^{*}=2 \lambda_{2} d_{1}^{*}, \\
d_{4}^{* 2}=\frac{2 \alpha c \lambda_{1} R_{0} d_{0}^{*}}{\lambda_{1}^{* 2} R_{0}^{*}}, \quad\left(1-J_{1}\right) d_{3}^{*}=2 \lambda_{2}^{*} d_{4}^{*}, \\
\Delta=\left(\frac{R_{0}^{*}}{R_{0}}\right)^{\frac{2}{2}} \operatorname{sgn}\left(\frac{\lambda_{1}}{\lambda_{1}^{*}}\right), \quad \hat{E}_{1}=\left(\frac{2 \lambda_{2}}{\lambda_{1}}+\frac{R_{1}}{R_{0}}\right), \quad \hat{E}_{2}=\left(\frac{2 \lambda_{2}^{*}}{\lambda_{1}^{*}}+\frac{R_{1}^{*}}{R_{0}^{*}}\right) \frac{R_{0} \lambda_{1}^{2}}{R_{0}^{*} \lambda_{1}^{* 2}} .
\end{gathered}
$$

Equations (5.15) must be solved subject to the following boundary conditions

$$
\begin{gathered}
Q_{L} \sim(1-J) \hat{Z}+\frac{\operatorname{Re}\left(A \exp \left(i \zeta^{*}\right)\right)}{\hat{Z}}, \quad \hat{Z} \rightarrow \pm \infty, \\
\Pi \sim \hat{Z}+\frac{\operatorname{Re}\left(A \exp \left(i \zeta^{*}\right)\right)}{\hat{Z}}, \quad \hat{Z} \rightarrow \pm \infty, \\
Q_{U} \sim\left(1-J_{1}\right) \hat{Z}_{1}+\frac{\operatorname{Re}\left(A \exp \left(i \zeta^{*}\right)\right)}{\hat{Z}_{1}}, \quad \hat{Z}_{1} \rightarrow \pm \infty, \\
\Phi \sim \hat{Z}_{1}+\frac{\operatorname{Re}\left(A \exp \left(i \zeta^{*}\right)\right)}{\hat{Z}_{1}}, \quad \hat{Z}_{1} \rightarrow \pm \infty,
\end{gathered}
$$

The normalized linear growth rate is $\kappa_{r}$ (defined below) so that $\operatorname{Re}\left(A_{\hat{X}} / A\right) \rightarrow \kappa_{r}$ as $\hat{X} \rightarrow-\infty$.

We note that the equations are now of a similar form to the evolution equations obtained by Goldstein and Wundrow (1990), and Gajjar (1994). The equations (5.15) are a generalization of those of Gajjar (1994) for flows with multiple critical layers. In our analysis however viscous effects have been ignored (such effects come into play when $h=O(R)^{-1 / 14}$ ), see Gajjar (1994). The equations are then modified to

$$
\begin{gathered}
\mathcal{L} Q_{L}-\gamma_{c} Q_{L \hat{Z} \hat{Z}}=J \operatorname{Re}\left(i A \exp \left(i \zeta^{*}\right)\right) \Pi_{\hat{Z}}+\gamma_{e} \Pi_{\hat{Z} \hat{Z}}, \\
\mathcal{L} \Pi-\gamma_{d} \Pi_{\hat{Z} \hat{Z}}=0, \\
\mathcal{L}_{\mathcal{U}} Q_{U}-\gamma_{c}^{*} Q_{U \hat{Z}_{1} \hat{Z}_{1}}=J_{1} \operatorname{Re}\left(i A \exp \left(i \zeta^{*}\right)\right) \Phi_{\hat{Z}_{2}}+\gamma_{e}^{*} \Phi_{\hat{Z}_{1} \hat{Z}_{1}}, \\
\mathcal{L}_{U} \Phi-\gamma_{d}^{*} \Phi_{\hat{Z}_{1} \hat{Z}_{1}}=0,
\end{gathered}
$$


where the operators $\mathcal{L}, \mathcal{L}_{\mathcal{U}}$ and the constants in (5.18) are given by

$$
\begin{gathered}
\mathcal{L} \equiv \frac{\partial}{\partial \hat{X}}+\hat{Z} \frac{\partial}{\partial \zeta^{*}}-\operatorname{Re}\left(i A \exp \left(i \zeta^{*}\right)\right) \frac{\partial}{\partial \hat{Z}} \\
\mathcal{L}_{u} \equiv \Delta \frac{\partial}{\partial \hat{X}}+\hat{Z}_{1} \frac{\partial}{\partial \zeta^{*}}-\operatorname{Re}\left(i A \exp \left(i \zeta^{*}\right)\right) \frac{\partial}{\partial \hat{Z}_{1}} \\
\gamma_{c}=\frac{S_{0} \mu\left(S_{0}\right) R^{-\frac{1}{2}} h^{-7}}{\lambda_{1} d_{1}^{* 3} \gamma}, \quad \gamma_{d}=\gamma_{c} / \operatorname{Pr}, \\
\gamma_{e}=\gamma_{d} J\left(\frac{\operatorname{Pr} \mu^{\prime}\left(S_{0}\right)}{R_{0} \mu\left(S_{0}\right)}-1\right), \quad \gamma_{c}^{*}=\frac{\mu\left(S_{0}^{*}\right) R_{0} \lambda_{1} d_{1}^{* 3}}{\mu\left(S_{0}\right) R_{0}^{*} \lambda_{1}^{*} d_{4}^{* 3}} \gamma_{c}, \\
\gamma_{d}^{*}=\gamma_{c}^{*} / \operatorname{Pr}, \quad \gamma_{e}^{*}=\gamma_{d}^{*} J_{1}\left(\frac{\operatorname{Pr} \mu^{\prime}\left(S_{0}^{*}\right)}{R_{0}^{*} \mu\left(S_{0}^{*}\right)}-1\right) .
\end{gathered}
$$

Here $\mu\left(T_{B}\right)$ is the viscosity law ( $S_{0}$ is defined in (2.4)), and because of the assumptions made in section $2, \gamma_{c}, \gamma_{c}^{*}$ take opposite signs.

The equations (5.18), with boundary conditions (5.17) govern the nonlinear evolution of the cross-flow vortex. Note that for a given basic flow the constants $J, J_{1}$ are fixed and the only free parameters are $\gamma_{c}$ and the wave-angle $\theta$. For the inviscid case $\gamma_{c}$ is zero.

\section{Linear Critical Layer}

The linear theory may be recovered from the above equations by first writing

$$
\begin{array}{cl}
Q_{L}=(1-J) \hat{Z}+\operatorname{Re}\left(\bar{Q}_{L} e^{i \zeta^{*}}\right), & Q_{U}=\left(1-J_{1}\right) \hat{Z}_{1}+\operatorname{Re}\left(\bar{Q}_{U} e^{i \zeta^{*}}\right), \\
\Pi=\hat{Z}+\operatorname{Re}\left(\bar{\Pi} e^{i \zeta^{*}}\right), & \Phi=\hat{Z}_{1}+\operatorname{Re}\left(\bar{\Phi} e^{i \zeta^{*}}\right),
\end{array}
$$

and then linearising the equations to give

$$
\begin{gathered}
\bar{Q}_{L \hat{X}}+i \hat{Z} \bar{Q}_{L}-\gamma_{c} \bar{Q}_{L \hat{z} \hat{z}}=i A+\gamma_{e} \bar{\Pi}_{\hat{z} \hat{z}}, \\
\Delta \bar{Q}_{U \hat{X}}+i \hat{Z}_{1} \bar{Q}_{U}-\gamma_{c}^{*} \bar{Q}_{U \hat{Z}_{1} \hat{z}_{1}}=i A+\gamma_{e}^{*} \bar{\Phi}_{\hat{Z}_{1} \hat{z}_{1}},
\end{gathered}
$$

The solution of these equations may be obtained by utilising the Fourier Transform technique of Hickernell (1984). This gives

$$
\begin{gathered}
\bar{\Pi}^{\dagger}=i A(\hat{X}+s) e^{\gamma_{d} s^{3} / 3} H\left(-s \operatorname{sgn}\left(\gamma_{d}\right)\right) \operatorname{sgn}\left(\gamma_{d}\right), \\
\bar{\Phi}^{\dagger}=i A(\hat{X}+\Delta s) e^{\gamma_{d}^{*} s^{3} / 3} H\left(-s \operatorname{sgn}\left(\gamma_{d}^{*}\right)\right) \operatorname{sgn}\left(\gamma_{d}^{*}\right), \\
\bar{Q}_{L}^{\dagger}=e^{\gamma_{c} s^{3} / 3} i A(\hat{X}+s)\left\{1-\frac{3 \gamma_{e}}{\left(\gamma_{d}-\gamma_{c}\right)}\left(1-\exp \left(\frac{\gamma_{d}-\gamma_{c}}{3} s^{3}\right)\right)\right\} H\left(-s \operatorname{sgn}\left(\gamma_{c}\right)\right) \operatorname{sgn}\left(\gamma_{c}\right),
\end{gathered}
$$




$$
\bar{Q}_{U}^{\dagger}=e^{\gamma_{c}^{*} s^{3} / 3} i A(\hat{X}+\Delta s)\left\{1-\frac{3 \gamma_{e}^{*}}{\left(\gamma_{d}^{*}-\gamma_{c}^{*}\right)}\left(1-\exp \left(\frac{\gamma_{d}^{*}-\gamma_{c}^{*}}{3} s^{3}\right)\right)\right\} \dot{H}\left(-s \operatorname{sgn}\left(\gamma_{c}^{*}\right)\right) \operatorname{sgn}\left(\gamma_{c}^{*}\right),
$$

where the Fourier Transforms are defined by

$$
U^{\dagger}(s)=\int_{-\infty}^{\infty} U \exp (-i s Z) d Z, \quad U(Z)=\frac{1}{2 \pi} \int_{-\infty}^{\infty} U^{\dagger} \exp (i s Z) d s,
$$

with $Z$ being $\hat{Z}$, or $\hat{Z}_{1}$ as appropriate. Also in $(5.20) H(x)$ denotes the Heaviside unit step function. The above solutions agree with those given in Leib (1991) for similar linear equations. The solutions (5.20) show that

$$
\frac{1}{\pi} \int_{0}^{2 \pi} \int_{-\infty}^{* \infty} Q_{L} e^{-i \zeta^{*}} d \zeta^{*} d \hat{Z}=i \pi \operatorname{sgn}\left(\gamma_{c}\right), \quad \frac{1}{\pi} \int_{0}^{2 \pi} \int_{-\infty}^{* \infty} Q_{U} e^{-i \zeta^{*}} d \zeta^{*} d \hat{Z}_{1}=i \pi \operatorname{sgn}\left(\gamma_{c}^{*}\right) .
$$

\section{Additional Comments and Discussion}

We may use the results in the previous section, or alternatively (5.13), (5.14) with the appropriate linear jumps, to obtain an expression for the linear growth rate of the cross-flow vortex. The scaled linear growth rate $\kappa_{r}$ is thus given by

$$
\kappa_{r}=\frac{R_{0} \lambda_{1} \gamma c \pi \cos \theta}{2}\left[\frac{1}{\lambda_{1}^{2} R_{0}}\left(\frac{2 \lambda_{2}}{\lambda_{1}}+\frac{R_{1}}{R_{0}}\right) \operatorname{sgn}\left(\lambda_{1}\right)+\frac{1}{\lambda_{1}^{* 2} R_{0}^{*}}\left(\frac{2 \lambda_{2}^{*}}{\lambda_{1}^{*}}+\frac{R_{1}^{*}}{R_{0}^{*}}\right) \operatorname{sgn}\left(\lambda_{1}^{*}\right)\right] .
$$

The expression (6.1) may also be expressed as

$$
\kappa_{r}=\frac{R_{0} \lambda_{1} \gamma \pi c \cos \theta}{2} \sum_{\mathrm{cl} l^{\prime s}}\left[\left(\frac{D\left(\rho_{B} D \bar{U}_{B}\right)}{\rho_{B}^{2} D\left(\bar{U}_{B}\right)^{3}}\right) \operatorname{sgn}\left(D \bar{U}_{B}\right)\right]
$$

where the summation is taken over all critical levels and the expressions inside the summation are evaluated at the critical levels, and $D \equiv \frac{\theta}{\partial Y}$.

As in Gajjar (1994) the unscaled wavenumber, frequency and growth rate terms are:

wavenumber $R^{\frac{1}{2}} \gamma_{*}=R^{\frac{1}{2}}(\gamma h+\ldots), \quad$ frequency $R^{\frac{1}{2}} \omega_{*}=R^{\frac{1}{2}}\left(\gamma h^{2} c \cos \theta+\ldots\right)$,

$$
\text { growthrate } R^{\frac{1}{2}} \kappa_{*}=R^{\frac{1}{2}}\left(\kappa_{r} h^{2}+\ldots\right) \text {. }
$$

Using (3.13),(4.5) the leading term in the expansion for the wavenumber is given by

$$
\gamma_{*}^{2}=\frac{\lambda_{1} R_{0} \omega_{*}}{U_{\infty}^{2}}\left(1-U_{\infty}^{2} M_{\infty}^{2}\right)^{\frac{1}{2}}
$$

The result (6.2) shows that for neutral waves we require

$$
\sum_{\mathrm{cl} ' s}\left[\left(\frac{D\left(\rho_{B} D \bar{U}_{B}\right)}{\rho_{B}^{2} D\left(\bar{U}_{B}\right)^{3}} \operatorname{sgn}\left(D \bar{U}_{B}\right)\right)=0\right.
$$


which is a generalization of the result that $D\left(\rho_{B} D U_{B}\right)=0$ for flows with a single critical level.

Unlike the planar case the result (6.1) shows a more complex dependence of the growth rate on the wave-angle $\theta$ since $\lambda_{1}, \lambda_{1}^{*}, \lambda_{2}, \lambda_{2}^{*}$ depend on $\theta$ through (2.5), (2.6). For the neutral case the condition that $\bar{U}_{B}=0$ together with (6.3) fixes the position of the critical level as well as $\theta$.

The second important observation stemming from (6.1) is as follows. For a twodimensional insulated, flat plate boundary layer with zero pressure gradient, the growth rate $\kappa_{r}$ is zero, Gajjar (1994). For a fully three-dimensional boundary layer this is no longer true because of the presence of the last two terms in (6.1). This shows that for the stability problem with cross-flow effects present, there is no real distinction between the insulated and non-insulated cases as the growth rates are of the same order in both cases.

The analysis used to derive (6.1) was based mostly on an inviscid analysis with viscous effects confined to the critical layers. The Stokes wall layer also contributes to the growth rate and as Appendix A shows this contribution can be written as

$$
R^{\frac{1}{2}} \kappa_{*}=R^{\frac{1}{2}} \kappa_{\tau} h^{2}+\ldots+R^{\frac{1}{2}} h^{-1} \frac{\gamma \lambda_{1}}{2 \sqrt{2}|c| \cos \theta}\left(\frac{\mu\left(S_{0}\right)}{R_{0}|c| \gamma \cos \theta}\right)^{\frac{1}{2}}+\ldots
$$

where the last term in (6.4) arises from the viscous wall layer. For $\theta$ not close to $\frac{\pi}{2}$ and $h>>R^{-\frac{1}{12}}$ the Stokes layer contribution in (6.4) is much smaller than the inviscid contribution. As $h$ decreases however the two terms become comparable when $h=O\left(R^{-\frac{1}{12}}\right)$. This implies an effective wavenumber of $O\left(R^{\frac{6}{12}}\right)$ and growth rates of $O\left(R^{\frac{1}{3}}\right)$. This is precisely the scaling for the upper-branch of a two-dimensional accelerating boundary layer in incompressible flow considered by Smith \& Bodonyi (1982), and extended to the threedimensional boundary layer case by Bassom \& Gajjar (1988). In this limit it is noted from (5.19) that $\gamma_{c}, \gamma_{c}^{*}$ are large parameters and as Appendix B shows, for $\left|\gamma_{c}\right|>>1$ the unsteady analysis remains largely intact but with the critical layers becoming strongly viscous critical layers of the Haberman (1972) type. Additional diffusion layers at the outer edges of the critical layers are necessary to reduce the large mean vorticity jump produced by the critical layers back to zero outside in the main part of the boundary layer. When $h=O\left(R^{-\frac{1}{12}}\right)$, from (5.19) it can be seen that $\gamma_{c}=O\left(R^{\frac{1}{12}}\right)$. From Appendix B the thickness of the critical layer is then of $O\left(R^{-\frac{1}{2}} h^{2} \gamma_{c}^{\frac{1}{3}}\right)=O\left(R^{-\frac{23}{36}}\right)$ which is the same as in Smith \& Bodonyi (1982). The diffusion layers at the outer edge of the critical layer have thickness $O\left(R^{-\frac{1}{2}} h^{2} \gamma_{c}^{\frac{2}{2}}\right)=O\left(R^{-\frac{15}{36}}\right)$. 
Hence in the formal limit when $h$ becomes of $O\left(R^{-\frac{1}{22}}\right)$ the linear growth rate is given by

$$
\begin{aligned}
R^{\frac{1}{2}} \kappa_{*} & =R^{\frac{1}{3}}\left(\frac{R_{0} \lambda_{1} \gamma c \pi \cos \theta}{2}\left[\frac{1}{\lambda_{1}^{2} R_{0}}\left(\frac{2 \lambda_{2}}{\lambda_{1}}+\frac{R_{1}}{R_{0}}\right) \operatorname{sgn}\left(\lambda_{1}\right)+\frac{1}{\lambda_{1}^{* 2} R_{0}^{*}}\left(\frac{2 \lambda_{2}^{*}}{\lambda_{1}^{*}}+\frac{R_{1}^{*}}{R_{0}^{*}}\right) \operatorname{sgn}\left(\lambda_{1}^{*}\right)\right]\right. \\
& \left.+\frac{\gamma \lambda_{1}}{2 \sqrt{2}|c| \cos \theta}\left(\frac{\mu\left(S_{0}\right)}{R_{0}|c| \gamma \cos \theta}\right)^{\frac{1}{2}}\right)+\ldots
\end{aligned}
$$

The condition for neutral waves is obtained by setting the expression given in (6.5) to zero and it can be seen that a special case of this result agrees with that obtained by Bassom \& Gajjar (1988) for incompressible flow. (The incompressible results are retrieved by setting $\rho_{B}$ to be constant).

When $h=O\left(R^{-\frac{1}{12}}\right)$ results for the unsteady nonlinear evolution of the cross-flow vortex may be similarly deduced. The appropriate expression for the leading term of the growth rate is then,

$$
R^{\frac{1}{3}}\left(-\frac{\gamma \lambda_{1} d_{1}^{*}}{\alpha c}\left[\hat{E}_{1} \operatorname{Im}\left(i \phi_{L}\right)+\hat{E}_{2} \operatorname{Im}\left(i \phi_{U}\right)\right]+\frac{\gamma \lambda_{1}}{2 \sqrt{2}|c| \cos \theta}\left(\frac{\mu\left(S_{0}\right)}{R_{0}|c| \gamma \cos \theta}\right)^{\frac{2}{2}}\right)+\ldots
$$

where the nonlinear jumps $\phi_{L}, \phi_{U}$ at the lower and upper critical layers respectively, are obtained from

$$
A \phi_{L}=\frac{1}{\pi} \int_{0}^{2 \pi} \int_{-\infty}^{* \infty} e^{-i \zeta^{*}} Q_{L} d \hat{Z} d \zeta^{*}, \quad A \phi_{U}=\frac{1}{\pi} \int_{0}^{2 \pi} \int_{-\infty}^{* \infty} e^{-i \zeta^{*}} Q_{U} \dot{d} \hat{Z} d \zeta^{*}
$$

and $Q_{L}, Q_{U}$ satisfy the steady versions of (5.19), (see also appendix B). Some results for $\phi_{L}$ for the single critical layer version of (5.19) and with the Chapman viscosity law are presented in Gajjar \& Cole (1989). For nonlinear neutral waves the expression in (6.6) is set to zero and this again extends the Bassom \& Gajjar (1988) results to the compressible case.

The full unsteady nonlinear problem governed by (5.17), (5.19) involving the solution of four coupled unsteady nonlinear critical layer equations poses a formidable numerical task and is not pursued here. The problem is being tackled using the methods, described in Gajjar (1994), for the single critical level case. 


\section{Summary}

In conclusion we have obtained the nonlinear equations governing the evolution of a long wavelength cross-flow vortex in a three-dimensional compressible boundary layer. These equations remain to be solved but the solution properties are of some interest especially in relation to the question of whether the cross-flow vortices attain an equilibrium amplitude or not. The one major simplifying assumption used in the present work is that the cross-Squire variations are small and negligible. The relaxation of this assumption requires further study but it is expected that as the $\bar{z}$ variations gradually increase the first major non-trivial influence will be felt in the critical layers. The question of shorter waves is addressed in Gajjar (1995) for the stationary cross-flow vortex where a novel integro-differential amplitude equation for the vortex has been obtained, and in Gajjar \& Arebi (1995) for the non-stationary case.

Although it has not been done here, it would be interesting to compare the predictions for the linear growth rate as given by (6.1-6.5) with full numerical solution of the compressible linear stability equations. Comparisons for the single critical layer case are shown in Gajjar (1994) and good agreement was found particularly for the insulated wall case. A special case of (6.3),(6.4) includes that for a two-dimensional boundary layer and it would be interesting to see how the viscous correction to the growth rate compares with the equivalent 'Orr-Sommerfeld' type or linear stability calculation for the first mode. The results (6.3),(6.4) obtained here are particularly useful as they provide an alternative route for investigating the effects of different parameters such as Mach number, wall heating, etc on the stability properties. Unlike the numerical solution of the linear stability equations, the analytic results given here may be used in a much more systematic and easier manner for investigating these different effects.

Finally we have shown how the unsteady critical layer analysis may be linked to the properties of the steady Haberman type of critical layer. In the limit that the Haberman parameter ( $\gamma_{c}$ as used in this work) becomes large, the major modification required in the unsteady critical layer structure is the inclusion of appropriate diffusion layers at the outer edges of the critical layers. The analysis in Appendix B apply equally to other flows including the accelerating boundary layer and channel flow as considered for example by Gajjar \& Smith (1985). An application of such ideas to channel flow with compliant boundaries is presented in Gajjar \& Sibanda (1995).

\section{Acknowledgement}

The author gratefully acknowledges the support of ICOMP, Nasa Lewis Research Centre, Cleveland where part of this work was done during the visits in 1992 and 1993. Dr's Goldstein, Hultgren, Leib and Wundrow are also thanked for many stimulating discussions on unsteady critical layers. A referee is also thanked for his comments. 


\section{Appendix A}

In this appendix we derive the correction to the inviscid growth rate arising from the Stokes wall layer. For the sake of clarity only the important details are given.

We first note that the thickness of the Stokes layer is given by

$$
y=R^{-\frac{3}{4}} h^{-1} \hat{y}, \quad \text { or } \quad Y=R^{-\frac{1}{4}} h^{-2} \hat{y}
$$

from a balance of the viscous and inertial terms in the Navier-Stokes equations. For the layer to be thin we require that $h \gg>R^{-\frac{1}{8}}$. Expansions for the flow quantities are readily written down using (3.4),(3.5) and in particular we have

$$
\begin{aligned}
\bar{u} & =R^{-\frac{1}{4}} h^{-1} \lambda_{1} \hat{y}+\delta \hat{u}_{1}+\ldots \\
v & =\delta R^{-\frac{1}{4}} \hat{v}_{1}+\ldots \\
\bar{w} & =\delta_{1} R^{-\frac{1}{4}} h^{-1} \hat{y}+\delta R^{-\frac{1}{4}} h^{-2} \hat{w}_{1}+\ldots \\
p & =p_{B}+\delta h \hat{p}_{1}+\ldots \\
\rho & =R_{0}+\delta R^{-\frac{1}{4}} h^{2} \hat{\rho}_{1}+\ldots
\end{aligned}
$$

Substitution into the Navier-Stokes equations yields the usual equations for the Stokes wall layer. These are easily solved and the finite part of the normal velocity component $\hat{v}_{1}$ as $\hat{y} \rightarrow \infty$, say $\hat{v}_{1 f}$, is calculated to be

$$
\hat{v}_{1 f}=\frac{\hat{p}_{1 \zeta}}{m \alpha c R_{0}}=\frac{\lambda_{1} \bar{A}_{0 \zeta}}{m}
$$

where

$$
m=\left(\frac{R_{0} \alpha|c| \gamma}{\mu\left(S_{0}\right)}\right)^{\frac{1}{2}} e^{-i \frac{\pi}{4} \operatorname{sgn}(c)}
$$

This shows that the expansion for the normal velocity in zone $I_{ \pm}$is of the form

$$
v=\delta\left(h^{2} \bar{v}_{1}+h^{3} \bar{v}_{2}+\ldots+R^{-\frac{1}{4}} \bar{v}_{s i}+\ldots\right),
$$

where we require

$$
\bar{v}_{s i}(Y=0)=\hat{v}_{1 f}
$$

To find the effect of the displacement (A3) on the growth rate it is necessary to introduce an additional multiple-scale coordinate $X_{2}$ defined by

$$
\frac{\partial}{\partial x} \rightarrow R^{\frac{1}{3}}\left(\alpha h \frac{\partial}{\partial \zeta}+\alpha h^{2} \frac{\partial}{\partial X}-\beta h \frac{\partial}{\partial \bar{z}}\right)+R^{\frac{1}{4}} \alpha h^{\nu+1} \frac{\partial}{\partial X_{2}}
$$




$$
\frac{\partial}{\partial z} \rightarrow R^{\frac{1}{2}}\left(\beta h \frac{\partial}{\partial \zeta}+\beta h^{2} \frac{\partial}{\partial X}+\alpha h \frac{\partial}{\partial \bar{z}}\right)+R^{\frac{1}{4}} \beta h^{\nu+1} \frac{\partial}{\partial X_{2}}
$$

where $\nu$ is a constant to be found. We now regard $\bar{A}_{0}$ as a function of $X_{2}$ also so that $\tilde{A}_{0}=\left(A\left(X, X_{2}\right) \exp (i \zeta)+c . c\right)$.

The expansions in the various regions are also modified so that, for example, in the $Y=O(1)$ region we have

$$
\begin{aligned}
& \bar{u}=U_{B}+\delta\left(\bar{u}_{1}+h \bar{u}_{2}+\ldots+R^{-\frac{1}{4}} h^{\nu} \bar{u}_{s i}+\ldots\right), \\
& v=\delta\left(h \tilde{v}_{1}+h^{2} \bar{v}_{2}+\ldots+R^{-\frac{1}{4}} h^{\nu+1} \bar{v}_{s i}+\ldots\right),
\end{aligned}
$$

and in region $I_{ \pm}$we have

$$
\begin{aligned}
& \bar{u}=h \lambda_{1} \bar{Y}+\ldots+\delta\left(\bar{u}_{1}+\ldots+R^{-\frac{1}{4}} h^{\nu} \bar{u}_{s i}+\ldots\right) \\
& v=\delta h^{2}\left(\bar{v}_{1}+\ldots+R^{-\frac{1}{4}} \bar{v}_{s i}+\ldots\right) \\
& p=p_{B}+\delta\left(h \bar{p}_{1}+\ldots+R^{-\frac{1}{4}} h^{-1} \bar{p}_{s i}+\ldots\right)
\end{aligned}
$$

For the solutions to match we require $\nu=-2$. Substitiution of $(A 6),(A 7)$, and similar expansions in the other regions, into the Navier-Stokes equations leads to a sequence of linear equations for the ' $s i$ ' quantities which are easily solved. For example

$$
\bar{v}_{s i}=-\frac{1}{R_{0} \lambda_{1}}\left(\bar{p}_{s i \zeta}+\bar{p}_{1 X_{2}}\right)-\frac{\alpha c}{\lambda_{1}} \bar{u}_{1 X_{2}}-\left(\lambda_{1} \bar{Y}-c\right) B s i
$$

and so on. Solving for each of the 'si' components in turn and matching then leads to

$$
\bar{p}_{s i}=\frac{\rho_{\infty} \gamma B_{s i} U_{\infty}^{2}}{\Omega}
$$

Hence setting $\bar{Y}=0$ in (A8) and using (A4),(A5),(4.4),(4.5) shows that

$$
A_{0 X_{2}}=-\frac{i \lambda_{1} \gamma}{2 c m \alpha} A_{0}
$$

This gives the correction to the growth rate as stated in (6.4). 


\section{Appendix B}

In this appendix we consider the solution of the system (5.18) with boundary conditions (5.16) in the limit as $\left|\gamma_{c}\right|$ becomes large. Without loss of generality we will take $\gamma_{c}$ to be positive and write $\gamma_{e}=e_{1} \gamma_{c}$, where $e_{1}$ is a constant. It is sufficient to consider just the lower critical layer as the analysis for the upper critical layer follows analogously. Hence the governing equations (with some of the suffices dropped) are

$$
\begin{gathered}
\frac{\partial Q}{\partial \hat{X}}+Z \frac{\partial Q}{\partial \zeta^{*}}-\operatorname{Re}\left(i A \exp \left(i \zeta^{*}\right)\right) \frac{\partial Q}{\partial Z}-\gamma_{c} \frac{\partial^{2} Q}{\partial Z^{2}}=J \operatorname{Re}\left(i A \exp \left(i \zeta^{*}\right)\right) \frac{\partial \Pi}{\partial Z}+e_{1} \gamma_{c} \frac{\partial^{2} \Pi}{\partial Z^{2}} \\
\frac{\partial \Pi}{\partial \hat{X}}+Z \frac{\partial \Pi}{\partial \zeta^{*}}-\operatorname{Re}\left(i A \exp \left(i \zeta^{*}\right)\right) \frac{\partial \Pi}{\partial Z}-\frac{\gamma_{c}}{P_{r}} \frac{\partial^{2} \Pi}{\partial Z^{2}}=0
\end{gathered}
$$

with boundary conditions

$$
\begin{gathered}
Q \sim(1-J) Z+\frac{\operatorname{Re}\left(A \exp \left(i \zeta^{*}\right)\right)}{Z}+\ldots \quad Z \rightarrow \pm \infty, \\
\Pi \sim Z+\frac{\operatorname{Re}\left(A \exp \left(i \zeta^{*}\right)\right)}{Z}+\ldots \quad Z \rightarrow \pm \infty .
\end{gathered}
$$

For $\gamma_{c}>>1$ there are two distinct regions which occur, see Figure 4. A balance of $Z Q_{\zeta^{*}}$ term with the viscous term shows that $Z=O\left(\gamma_{c}^{\frac{1}{3}}\right)$. This region, which we will denote by CL1, comprises the main part of the critical layer in which the solution properties are similar to those for a Haberman type of steady (or equilibrium) critical layer. A balance of the $Q_{\hat{X}}$ term with the viscous term shows that $Z=O\left(\gamma_{c}^{\frac{1}{2}}\right)$, and we will denote this region by the label D1. One of the important properties of the Haberman critical layer in region CL1 is that it generates a mean vorticity jump, see for instance Stewartson (1981). The main function of the region D1 here, or diffusion layer to use a more familiar terminology, is to restore the mean velocity jump to zero outside the critical layer as required by (B2).

Thus in region CL1 we set $Z=\gamma_{c}^{\frac{1}{3}} Z_{1}, A=\gamma_{c}^{\frac{2}{3}} \bar{A}$ and we have the expansions

$$
Q=\bar{Q}_{0}+\ldots, \quad \Pi=\bar{\Pi}_{0}+\ldots
$$

Substitution into (B1),(B2) then shows that at leading order $\bar{Q}_{0}, \bar{\Pi}_{0}$ satisfy

$$
\begin{gathered}
Z_{1} \frac{\partial \bar{Q}_{0}}{\partial \zeta^{*}}-\operatorname{Re}\left(i \bar{A} \exp \left(i \zeta^{*}\right)\right) \frac{\partial \bar{Q}_{0}}{\partial Z_{1}}-\frac{\partial^{2} \bar{Q}_{0}}{\partial Z_{1}^{2}}=J \operatorname{Re}\left(i \bar{A} \exp \left(i \zeta^{*}\right)\right) \frac{\partial \bar{\Pi}_{0}}{\partial Z_{1}}+e_{1} \frac{\partial^{2} \bar{\Pi}_{0}}{\partial Z_{1}^{2}} \\
Z_{1} \frac{\partial \bar{\Pi}_{0}}{\partial \zeta^{*}}-\operatorname{Re}\left(i \bar{A} \exp \left(i \zeta^{*}\right)\right) \frac{\partial \bar{\Pi}_{0}}{\partial Z_{1}}-\frac{\partial^{2} \bar{\Pi}_{0}}{\partial Z_{1}^{2}}=0 .
\end{gathered}
$$


These are just the compressible version of the Haberman equations, see Gajjar \& Cole (1989), and they have the property that

$$
\begin{gathered}
\bar{Q}_{0} \sim(1-J) Z_{1}+H^{ \pm}(\hat{X})+\frac{\operatorname{Re}\left(\bar{A}(\hat{X}) \exp \left(i \zeta^{*}\right)\right)}{Z_{1}}+\ldots \quad Z_{1} \rightarrow \pm \infty, \\
\bar{\Pi}_{0} \sim Z_{1}+\Delta^{ \pm}(\hat{X})+\frac{\operatorname{Re}\left(\bar{A}(\hat{X}) \exp \left(i \zeta^{*}\right)\right)}{Z_{1}}+\ldots \quad Z_{1} \rightarrow \pm \infty .
\end{gathered}
$$

The quantity $\left(H^{+}-H^{-}\right)$is the mean vorticity jump produced by the critical layer. Note that in (B3),(B4), $\bar{A}, H^{ \pm}, \Delta^{ \pm}$are all dependent on $\hat{X}$, but since $\hat{X}$ appears only as a parameter these equations are solved exactly as in the steady case.

It is convenient to treat the diffusion layers above and below the critical layers simultaneously. Hence in region $\mathrm{D} 1^{ \pm}$, see Figure 3, we set $Z=\gamma_{c}^{\frac{1}{2}} \eta$ and (B4) imply the expansions

$$
\begin{aligned}
& Q=\gamma_{c}^{\frac{1}{6}} q_{0}+q_{1}+\gamma_{c}^{-\frac{1}{6}} q_{2}+\gamma_{c}^{-\frac{2}{6}} q_{3}+\gamma_{c}^{-\frac{3}{6}} q_{4}+\ldots, \\
& \Pi=\gamma_{c}^{\frac{1}{6}} \Pi_{0}+\Pi_{1}+\gamma_{c}^{-\frac{2}{6}} \Pi_{2}+\gamma_{c}^{-\frac{2}{6}} \Pi_{3}+\gamma_{c}^{-\frac{3}{6}} \Pi_{4}+\ldots
\end{aligned}
$$

Substitution into (B1) then yields a sequence of equations given by

$$
\begin{gathered}
\eta \Pi_{0 \zeta^{*}}=0, \quad \eta q_{0 \zeta^{*}}=0, \\
\eta \Pi_{1 \zeta^{*}}=0, \quad \eta q_{1 \zeta^{*}}=0, \\
\eta \Pi_{2 \zeta^{*}}-\operatorname{Re}\left(i \bar{A} e^{i \zeta^{*}}\right) \Pi_{0 \eta}=0, \quad \eta q_{2 \zeta^{*}}-\operatorname{Re}\left(i \bar{A} e^{i \zeta^{*}}\right) q_{0 \eta}=J \operatorname{Re}\left(i \bar{A} e^{i \zeta^{*}}\right) \Pi_{0 \eta}, \\
\eta \Pi_{3 \zeta^{*}}+\Pi_{0 \hat{X}}-\operatorname{Re}\left(i \bar{A} e^{i \zeta^{*}}\right) \Pi_{1 \eta}-\frac{1}{P r} \Pi_{0 \eta \eta}=0, \\
\eta q_{3 \zeta^{*}}+q_{0 \hat{X}}-\operatorname{Re}\left(i \bar{A} e^{i \zeta^{*}}\right) q_{1 \eta}-q_{0 \eta \eta}=J \operatorname{Re}\left(i \bar{A} e^{i \zeta^{*}}\right) \Pi_{1 \eta}+e_{1} \Pi_{0 \eta \eta}, \\
\eta \Pi_{4 \zeta^{*}}+\Pi_{1 \hat{X}}-\operatorname{Re}\left(i \bar{A} e^{i \zeta^{*}}\right) \Pi_{2 \eta}-\frac{1}{P r} \Pi_{1 \eta \eta}=0, \\
\eta q_{4 \zeta^{*}}+q_{1 \hat{X}}-\operatorname{Re}\left(i \bar{A} e^{i \zeta^{*}}\right) q_{2 \eta}-q_{1 \eta \eta}=J \operatorname{Re}\left(i \bar{A} e^{i \zeta^{*}}\right) \Pi_{2 \eta}+e_{1} \Pi_{1 \eta \eta} .
\end{gathered}
$$

These equations may be solved in series. First (B6),(B9) show that

$$
\Pi_{0}=\eta, \quad q_{0}=(1-J) \eta .
$$

The solution of (B7) gives

$$
\Pi_{1}=\Pi_{1 M}(\hat{X}, \eta), \quad q_{1}=q_{1 M}(\hat{X}, \eta) .
$$

The solution of (B8) yields

$$
\Pi_{2}=\frac{\operatorname{Re}\left(\bar{A} e^{i \zeta^{*}}\right)}{\eta}+\Pi_{2 M}(\hat{X}, \eta), \quad q_{2}=\frac{\operatorname{Re}\left(\bar{A} e^{i \zeta^{*}}\right)}{\eta}+q_{2 M}(\hat{X}, \eta) .
$$


If we substitute for $\Pi_{0}, q_{0}, \Pi_{1}, q_{1}, \Pi_{2}, q_{2}$ into (B10) and use (B11-B13), then after averaging (B10) over a period in $\zeta^{*}$ gives

$$
\frac{\partial \Pi_{1 M}}{\partial \hat{X}}-\frac{\partial^{2} \Pi_{1 M}}{\partial \eta^{2}}=0, \quad \frac{\partial q_{1 M}}{\partial \hat{X}}-\frac{\partial^{2} q_{1 M}}{\partial \eta^{2}}=e_{1} \frac{\partial^{2} \Pi_{1 M}}{\partial \eta^{2}} .
$$

To match with region CL1 we require that

$$
\Pi_{1 M}(\hat{X}, \pm 0)=\Delta^{ \pm}(\hat{X}), \quad q_{1 M}(\hat{X}, \pm 0)=H^{ \pm}(\hat{X}),
$$

The problem $(\mathrm{B} 14 \mathrm{a}, \mathrm{c})$ is identical to that solved by Smith et al.(1993) in their investigation of vortex wave interactions. The solution of (B14) may be obtained using Fourier transforms and it can be shown that the solutions which decay to zero at $\eta= \pm \infty$ are given by

$$
\Pi_{1 M}^{ \pm}=\frac{|\eta| \sqrt{P r}}{2 \sqrt{\pi}} \int_{-\infty}^{\hat{X}} \Delta^{ \pm}(s) \frac{\exp \left(-\frac{\eta^{2} P_{r}}{4(\hat{X}-s)}\right)}{(\hat{X}-s)^{\frac{3}{2}}} d s .
$$

If $P r=1$ then

$$
\begin{aligned}
q_{1 M}^{ \pm}=\int_{-\infty}^{\hat{X}} & \frac{|\eta|}{2 \sqrt{\pi}} H^{ \pm}(s) \frac{\exp \left(-\frac{\eta^{2}}{4(\hat{X}-s)}\right)}{(\hat{X}-s)^{\frac{3}{2}}} d s \\
& \quad-e_{1} \frac{|\eta|}{2 \sqrt{\pi}} \int_{-\infty}^{\hat{X}} \Delta^{ \pm}(\hat{X}-s) \frac{\partial}{\partial s}\left(\frac{H(s)}{s^{\frac{1}{2}}} \exp \left(\frac{-\eta^{2}}{4 s}\right)\right) d s
\end{aligned}
$$

and if $\operatorname{Pr} \neq 1$ then

$$
\begin{aligned}
q_{1 M}=\frac{\mid \eta}{2 \sqrt{\Pi}} \int_{-\infty}^{\hat{X}} & {\left[\left(H^{ \pm}(s)+\frac{e_{1} \Delta^{ \pm}(s)}{(P r-1)}\right) \frac{\exp \left(-\frac{\eta^{2}}{4(\hat{X}-s)}\right)}{(\hat{X}-s)^{\frac{3}{2}}}\right.} \\
& \left.-\frac{e_{1} \Delta^{ \pm}(s) \sqrt{P_{r}}}{(P r-1)} \frac{\exp \left(-\frac{\eta^{2} P_{r}}{4(\hat{X}-s)}\right)}{(\hat{X}-s)^{\frac{3}{2}}}\right] d s .
\end{aligned}
$$

The \pm refers to the solutions in the regions $\mathrm{D}^{ \pm}$above and below the regions $\mathrm{CLI}$ respectively. 


\section{References}

Arnal, D., (1986), Three-dimensional boundary layers: laminar-turbulent transition. AGARD Rep. no. 741.

Balakumar, P., \& Malik, M.R., (1990), Travelling disturbances in rotating disk flow. Theor. Comp. Fluid Dyn., 2, 125.

Bassom, A.P., \& Gajjar, J.S.B., (1988), Non-stationary cross-flow vortices in a threedimensional boundary layer. Proc. Roy. Soc., A 417, 179.

Bippes, H., \& Nitchke-Kowsky, P., (1987) Experimental study of instability modes in a three-dimensional boundary layer. A.I.A.A. pap no. 87-1336.

Cowley, S.J., \& Wu, X., (1994), Asymptotic approaches to transition modelling. AGARD Rep. no 793, Chap. 3, 1-38.

Corke, T.C., \& Mangano, R.A., (1989), Resonant growth of three-dimensional modes in transitioning Blasius layers. J. Fluid Mech., 209, 93.

Gajjar, J.S.B., (1994), Nonlinear evolution of a first mode oblique wave in a compressible boundary layer. Part I Heated/cooled walls. I.M.A.J. Appl. Math., 53, $221 .$.

Gajjar, J.S.B., (1995), Nonlinear development of a stationary-cross flow vortex, (submitted to J. Fluid Mech.)

Gajjar, J.S.B., \& Arebi, M., (1995). Nonlinear spatial/temporal development of nonstationary cross-flow vortices, (in preparation).

Gajjar, J.S.B., \& Cole, J.W., (1989), Upper branch stability of compressible boundary layer flows. Theo. Comput. Fluid Dyn., 1105.

Gajjar, J.S.B., \& Smith, F.T., (1985), On the global instability of free disturbancs with a time-dependent nonlinear viscous critical layer. J. Fluid Mech., 157, 53.

Gajjar, J.S.B., \& Sibanda, P., (1995), The hydrodynamic stability of channel flow with compliant boundaries, (to appear in Theo. Comp. Fluid Dyn.).

Goldstein, M.E., (1994), Nonlinear interactions between oblique instability waves on nearly parallel shear flows. Physics of Fluids, 6, (2), 724.

Goldstein, M.E., \& Choi, S.W., (1989), Nonlinear evolution of interacting oblique waves on two-dimensional shear layers. J. Fluid Mech., 207, 97. Also Corrigendum, J. Fluid Mech., (1990), 216, 659.

Goldstein,M.E., Durbin, P.A., \& Leib, S.J., (1987), Roll-up of vorticity in an adverse pressure gradient boundary layers. J. Fluid Mech., 183, 325.

Goldstein, M.E., \& Hultgren, L.S., (1988), Nonlinear spatial evolution of an externally excited instability wave in a free shear layer. J. Fluid Mech., 197, 295.

Goldstein, M.E., \& Leib, S.J., (1988), Nonlinear roll-up of externally excited free shear layers. J. Fluid Mech., 191, 481.

Goldstein.M.E., \& Lee, S.S., (1992), Fully coupled resonant-triad interaction in an adverse pressure-gradient boundary layer. J. Fluid Mech., 245, 523. 
Goldstein, M.E., \& Wundrow, D.W., (1990), Spatial evolution of nonlinear acoustic mode instabilities on hypersonic boundary layers. J. Fluid Mech., 219, 585.

Gregory, N., Stuart, J.T., \& Walker, W.S., (1955), On the instability of three-dimensional boundary layers with application to the flow due to a rotating disk. Phil. Trans. Roy. Soc., A 248, 155.

Haberman, R., (1972), Critical layers in parallel flows. Stud. Appl. Math., 51, 139.

Hall, P., (1986), An asymptotic investigation of the stationary modes of instability of the boundary layer on a rotating disk. Proc. Roy. Soc. Lond., A 406, 93.

Hall, P. \& Smith, F.T., (1991), On strongly nonlinear vortex/wave interactions in boundarylayer transition. J. Fluid Mech., 245, 641.

Hickernell, F.J., (1984), Time-dependent critical layers in shear flows on the beta-plane. J. Fluid Mech., 142, 431.

Hultgren, L.S., (1992), Nonlinear spatial equilibration of an externally excited instability wave in a free shear layer. J. Fluid Mech., 236, 635.

Kachanov, Y.S., \& Levchenko, V,Y., (1984), The resonant interaction of disturbances at laminar-turbulent transition in a boundary layer. J. Fluid Mech., 138, 209.

Klebanoff, P.S., Tidstrom, K.D., \& Sargent, L.M., (1962), The three-dimensional nature of boundary-layer instability. J. Fluid Mech., 12, 1.

Kohama, Y., Saric, W.S., \& Hoos, J.A., (1991), A high-frequency secondary instability of cross-flow vortices that leads to transition. in Proc. Royal Aero. Soc. conf. on Boundary Layer Transition and Control, Cambridge. 4.1.

Leib, S.J., (1991), Nonlinear evolution of subsonic and supersonic disturbances on a compressible mixing layer. J. Fluid Mech., 224, 551.

Lees, L., \& Lin, C.C., (1946), NASA Tech. Note No. 1115.

Mack, L.M., (1984), AGARD Tech. Rep no. 709.

Mack, L.M., (1986), in Stability of time-dependent and spatially varying flows,

(ed D.L.Dwoyer and M.Y. Hussaini), Springer.

Malik, M., (1986), The neutral curve for stationary disturbances in rotating disk flow. J. Fluid Mech., 164, 275.

Malik, M., \& Li, F., (1992), Three-dimensional boundary layer stability and transition. S.A.E. Tech Pap. no 921991.

Malik, M., Li, F., \& Chang, C.L., (1994), Crossflow disturbances in three-dimensional boundary layers: nonlinear development, wave interaction and secondary instability, J. Fluid Mech., 268, 1.

Maslowe, S. A:, (1986), Critical layers in shear flows. Ann. Rev. Fluid Mech., 18, 405.

Meyer, F., \& Kleiser, L., (1988), Numerical investigation of transition in $3 D$ boundary layers. Proc. AGARD Sysmposium on Fluid Dynamics of Three-Dimensional Turbulent Shear FLows and Transition, Cesme, Turkey. 
Muller, B., \& Bippes, H., (1988), Experimental study of instability modes in a threedimensional boundary layer. Proc. AGARD Sysmposium on Fluid Dynamics of ThreeDimensional Turbulent Shear FLows and Transition, Cesme, Turkey.

Nishioka, M., Asai, M., \& Iida, S., (1979), in Laminar Turbulent Transition. IUTAM meeting Stuttgart.

Poll, D.I.A., (1985), Some observations of the transition process on the windward face of a long yawed cylinder. J. Fluid Mech., 150, 329.

Reed, H.L., \& Saric, W.S., (1989), Stability of three-dimensional boundary layers. Ann. Rev. Fluid Mech., 21235.

Smith, F.T., \& Bodonyi, R.J., (1982), Nonlinear critical layers and their development in streaming flow stability. J. Fluid Mech., 118, 165.

Smith, F.T., \& Bowles, (1992), Transition theory and experimental comparisons on (I) amplification into streets and (II) a strongly nonlinear break-up criterion. Proc. Roy. Soc. Lond., A 439, 163.

Smith, F.T., Brown,S.N., Brown, P., (1993), Initiation of three-dimensional nonlinear transition paths from an inflexional profile. Euro. J. Fluid Mech., 12, 447.

Smith, F.T., \& Stewart, P.A., (1987), The resonant-triad nonlinear interaction in boundary layer transition. J. Fluid Mech., 179, 227.

Stewart, P., \& Smith, F.T., (1992), Development of three-dimensional nonlinear blow-up from a nearly planar initial disturbance, in boundary layer transition. J. Fluid Mech., 244, 79.

Stewartson, K., (1981), Marginally stable inviscid flows with critical layers. I.M.A.J. App. Math., 27, 133.

Wilkinson, S.P., \& Malik, M.R., (1983), Stability experiments in rotating disk flow. AIAA Pap. no. 83-1760.

Wundrow, D.M, (1989), Ph.D. Thesis, State Univ. of New York at Buffalo.

$\mathrm{Wu}, \mathrm{X} .$, (1993), Nonlinear temporal-spatial modulation of near-planar Rayleigh waves in shear flows: formation of streamwise vortices. J. Fluid Mech., 256., 684.

$\mathrm{Wu}$, X., Lee, S.S., \& Cowley, S.J., (1993), On the weakly nonlinear three-dimensional instability of shear flows to pairs of oblique waves: the Stokes layer as a paradigm. J. Fluid Mech., 253, 681.

Wu, X., Stewart, P.A., \& Cowley, S.J., (1994), On the weakly nonlinear development of Tollmien Schlichting wave-trains in boundary layers. Submitted to J. Fluid Mech. 


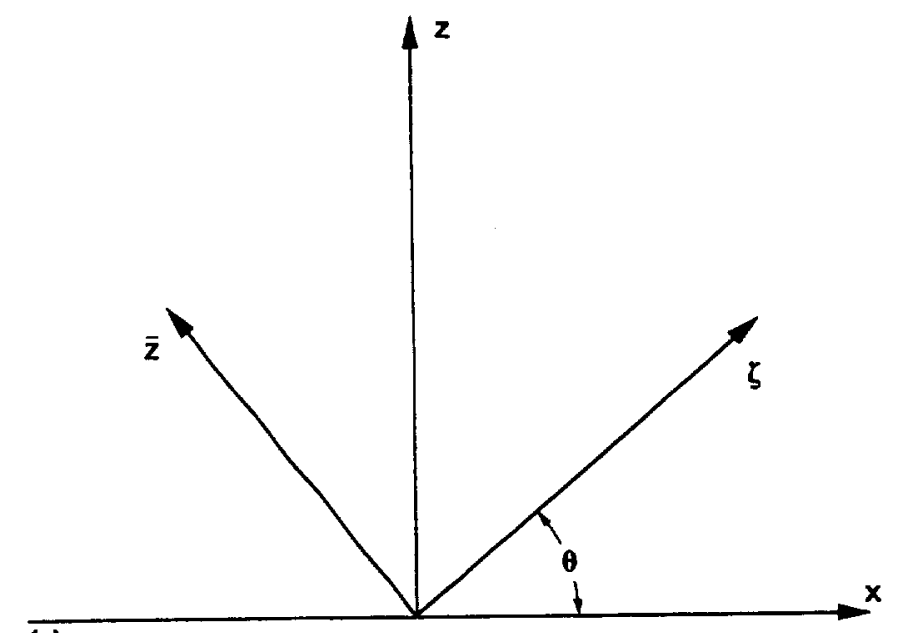

(a)

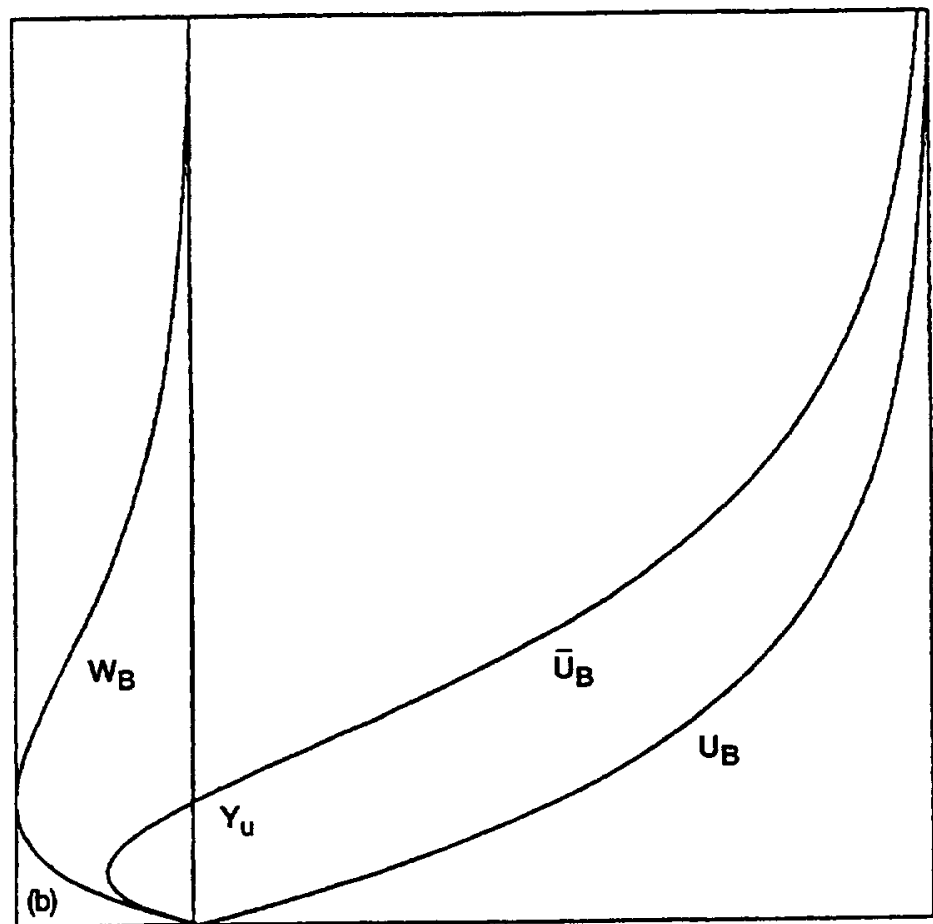

Figure 1. $-U_{B}, W_{B}, \bar{U}_{B}$ are as in the text. $Y_{U}$ is the location of the upper critical layer. (a) A sketch showing the coordinate system with the Squire coordinates $\bar{\zeta}$ and $\bar{z}$. (b) A sketch of typical velocity profiles for a three-dimensional boundary layer. 


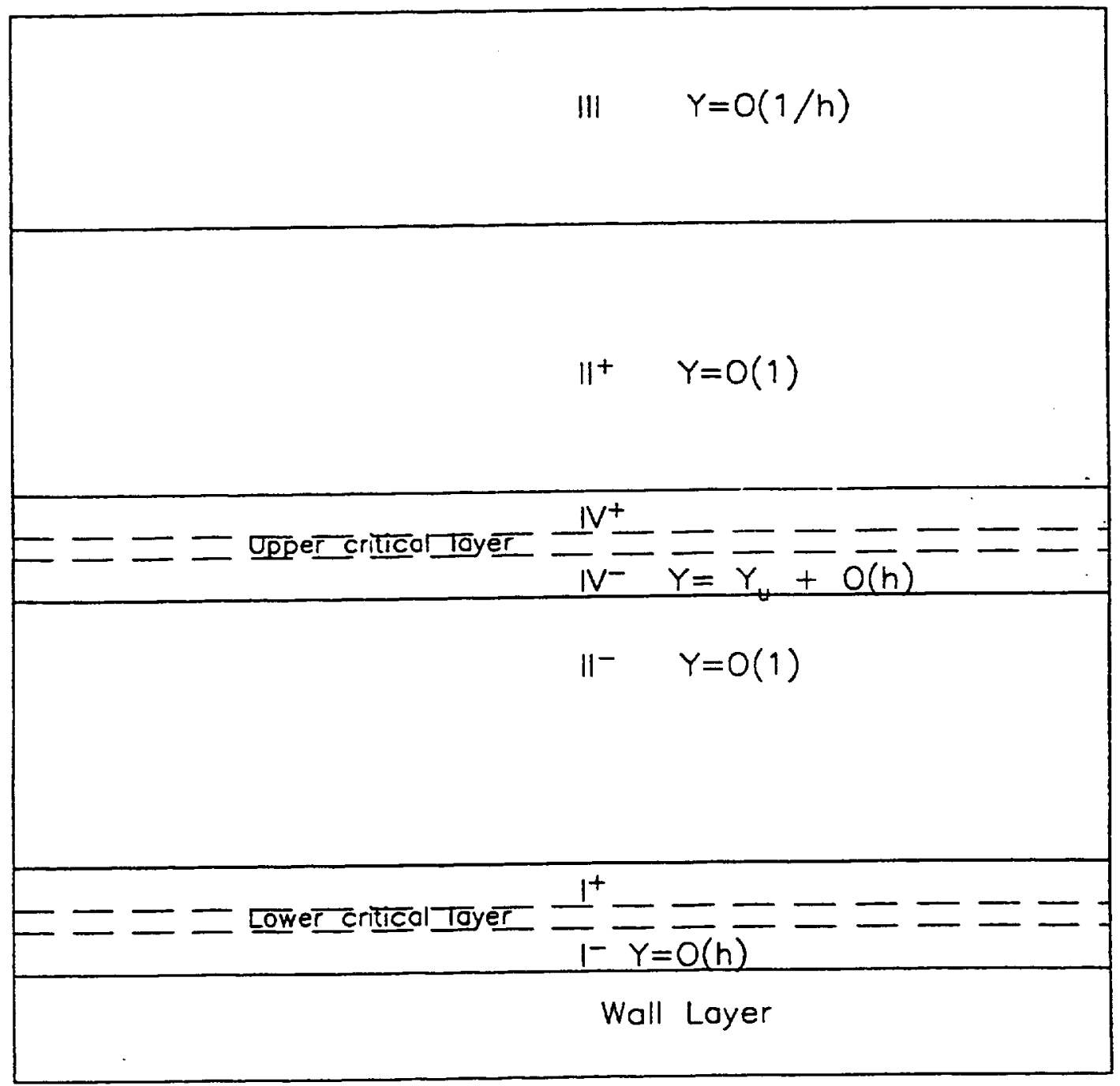

Figure 2.-A schematic diagram showing the various regions of the flow including the critical layers. 


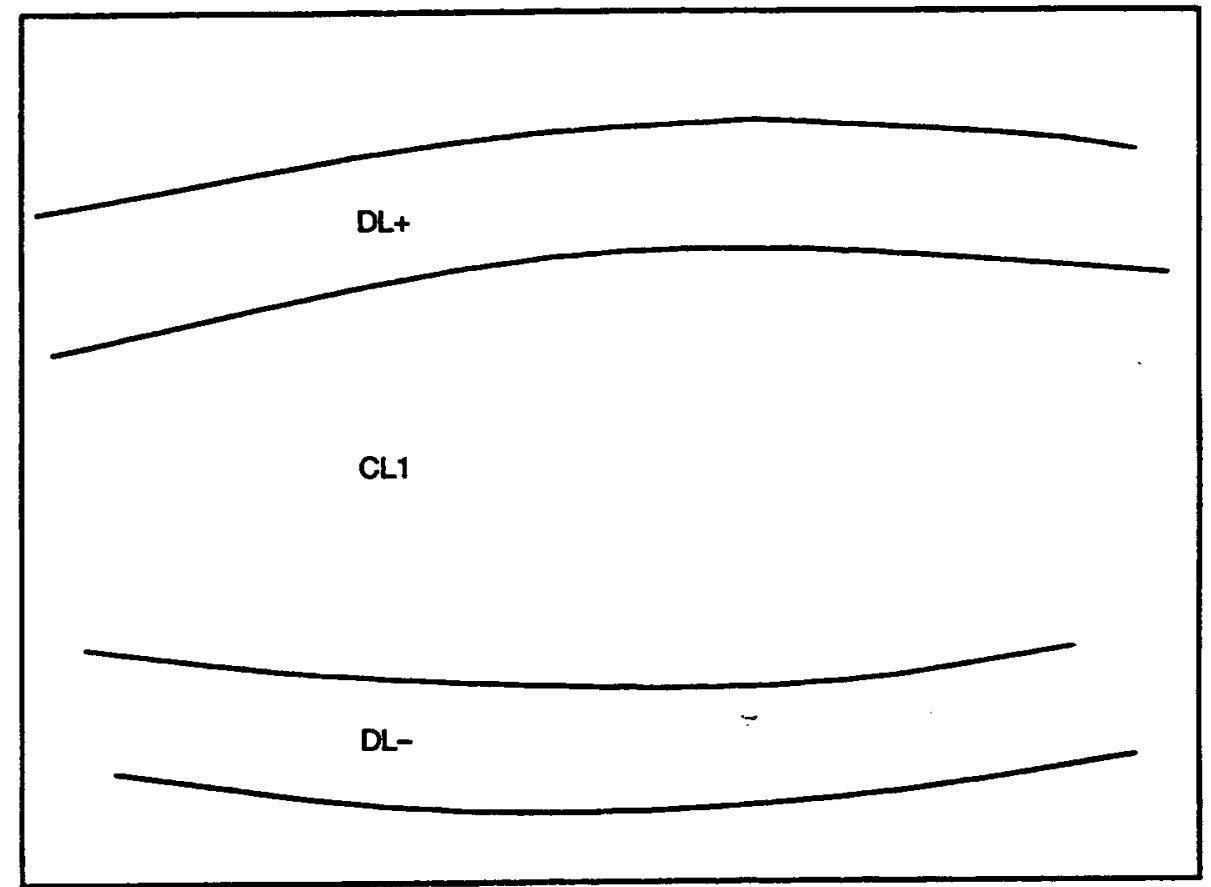

Figure 3.-A sketch of the diffusion layers $\mathrm{DL}^{ \pm}$, and the inner critical layer region CL1. 


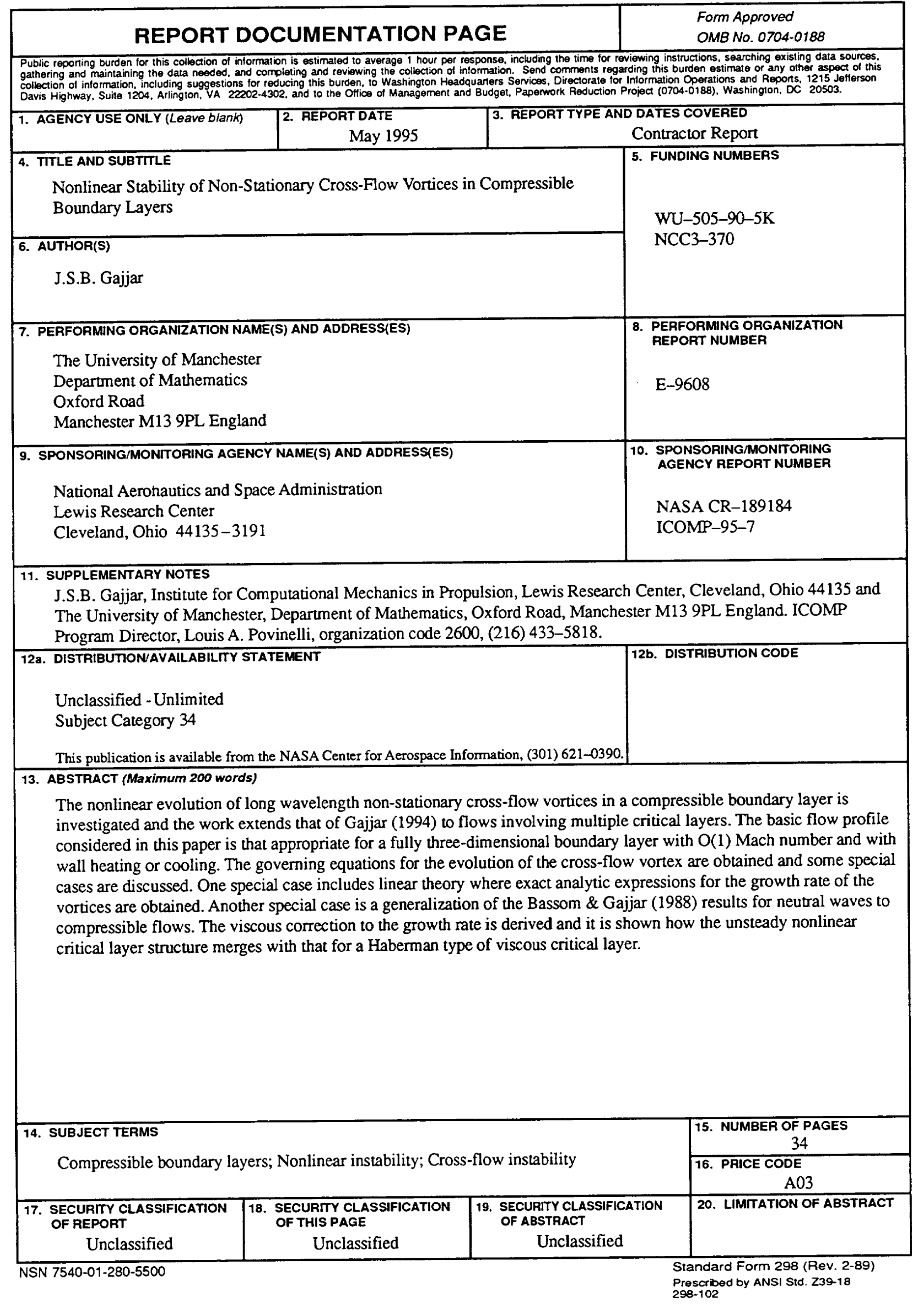


. 